\title{
The log-canonical threshold of a plane curve
}

\author{
By CARLOS GALINDO, FERNANDO HERNANDO \\ Instituto Universitario de Matemáticas y Aplicaciones de Castellón \\ and Departamento de Matemáticas, \\ Universitat Jaume I, Campus de Riu Sec. 12071 Castelló (Spain) \\ e-mail: galindo@mat.uji.es; carrillf@mat.uji.es \\ AND FRANCISCO MONSERRAT \\ Instituto Universitario de Matemática Pura y Aplicada, \\ Universidad Politécnica de Valencia, \\ Camino de Vera s/n, 46022 Valencia (Spain) \\ e-mail: framonde@mat.upv.es \\ (Received ; revised )
}

\section{Abstract}

We give an explicit formula for the log-canonical threshold of a reduced germ of plane curve. The formula depends only on the first two maximal contact values of the branches and their intersection multiplicities. We also improve the two branches formula given in [27].

\section{Introduction}

Let $X_{0}$ be a nonsingular variety over an algebraically closed field $k$ and consider a nonzero ideal sheaf $\mathfrak{a} \subseteq \mathcal{O}_{X_{0}}$. Assume the existence of a log resolution $\pi: X \rightarrow X_{0}$ of $\mathfrak{a}$ and let $F$ be the effective divisor such that $\mathfrak{a} \mathcal{O}_{X}=\mathcal{O}_{X}(-F)$. For any rational number $t>0$, the multiplier ideal sheaf $\mathcal{J}\left(\mathfrak{a}^{t}\right)$ is defined to be $\mathcal{J}\left(\mathfrak{a}^{t}\right)=\pi_{*} \mathcal{O}_{X}\left(K_{X \mid X_{0}}-\lfloor t F\rfloor\right)$, where $K_{X \mid X_{0}}$ denotes the relative canonical divisor of $\pi$ (that is, the unique exceptional divisor on $X$ such that $\mathcal{O}_{X}\left(K_{X \mid X_{0}}\right)$ is the dual of the relative jacobian sheaf [30, page $\left.206(2.3)\right]$ ) and $\lfloor\cdot\rfloor$ the round-down or the integer part of the corresponding divisor. A similar definition can be given for divisors on $X_{0}$. Multiplier ideals have been introduced and studied for complex varieties and admit an interesting analytic setting but the definition we use only depends on the existence of a log resolution. Multiplier ideals have a precedent, adjoint ideals which were introduced by Lipman in [29], and [28, Chapters 9, 10, 11] is the main reference for them. They are an important tool in birational geometry and singularity theory. Among other reasons, it is worthwhile to mention that they provide information on the type of singularity corresponding to the ideal $\mathfrak{a}$ and are very useful because accomplish several vanishing theorems. However, explicit computations are hard since they involve either to compute resolutions of singularities or to obtain difficult integrals.

Attached to $\mathfrak{a}$, there exists an increasing sequence of rational numbers $0=\iota_{0}<\iota_{1}<$ $\iota_{2}<\cdots$, called jumping numbers of $\mathfrak{a}$, such that $\mathcal{J}\left(\mathfrak{a}^{j}\right)=\mathcal{J}\left(\mathfrak{a}^{\iota_{l}}\right)$ for $\iota_{l} \leqslant j<\iota_{l+1}$ 
and $\mathcal{J}\left(\mathfrak{a}^{\iota^{l+1}}\right) \subset \mathcal{J}\left(\mathfrak{a}^{\iota^{l}}\right)$ for each $l \geqslant 0$. That is, the family of multiplier ideals of $\mathfrak{a}$ is totally ordered by inclusion and parameterized by non-negative rational numbers (see [17] for more information about the antecedents of these numbers). Computing jumping numbers is not easy. They are known for analytically irreducible (germs of) plane curves (see $[\mathbf{2 4}, \mathbf{3 7}, \mathbf{3 2}]$ and there exist algorithms for obtaining them for ideals of the local ring at a rational singularity of a complex algebraic surface $[\mathbf{3 6}, \mathbf{1}]$. A combinatorial criterium for a rational number to be a jumping number of a complete ideal $\mathfrak{a}$ of finite co-length in a local regular 2-dimensional ring is given in [22]. The case when $\mathfrak{a}$ is simple is well-known since jumping numbers and Poincaré series (an algebraic object relating jumping numbers and multiplier ideals) have been computed $[\mathbf{2 4}, \mathbf{1 9}]$, see also [2] for the Poincaré series in the non-simple case.

The first non-zero jumping number $\iota_{1}$ is named the log-canonical threshold of $\mathfrak{a}$. This number is, possibly, the most interesting of the jumping numbers; it appears in many different contexts and is related with rather different objects. Indeed, if $\mathfrak{a}$ is given by a polynomial providing a complex hyper-surface germ with an isolated singularity, the logcanonical threshold can be computed, theoretically, via, the $L^{2}$ condition for holomorphic functions [28], the growth of the codimension of jet schemes [31], the poles of the motivic zeta function $[\mathbf{2 0}]$, the generalized and twisted Bernstein-Sato polynomial $[\mathbf{2 5}, \mathbf{1 1}]$, the test ideals [21], the Arnold's complex oscillation index [25], the Hodge spectrum [9] and, as we have said, the orders of vanishing on a log resolution.

It seems that the first (implicit) use of the log-canonical threshold was in [5] where a conjecture of Gelfand was proved. In the 1980's this number was seen as one of the invariants considered by Steenbrink [35] in the so-called spectrum of a singularity. It was named complex singularity exponent and some of its properties were proved by Varchenko $[\mathbf{3 8}, 39,40]$. Afterwards, Shokurov $[\mathbf{3 3}]$ considered the log-canonical threshold within the context of birational geometry. This concept allows us to define log-canonical pairs which play a fundamental role in the Minimal Model Program that recently has achieved a great advance [7]. The paper [6] on the Shokurov conjecture (on the ACC for logcanonical thresholds of non-necessarily nonsingular ambient spaces) has been crucial for that progress.

Despite of being a very interesting number, only a few explicit generic computations of log-canonical thresholds are known. For instance, as we have mentioned, it is known for analytically irreducible (germs of) plane curves over algebraically closed fields. It is $\frac{1}{\beta_{0}}+\frac{1}{\beta_{1}}$, where $\left(\bar{\beta}_{0}, \bar{\beta}_{1}\right)$ are the first two maximal contact values of the curve $[\mathbf{2 3}, \mathbf{2 4}]$. In the complex case, the log-canonical thresholds of irreducible quasi-ordinary hypersurface singularities are also known [10] and the motivic zeta function was used for this computation. However, there is no formula for computing the log-canonical threshold of a reduced plane curve. This is the goal of this paper. Uniquely, in the complex case, a formula for the two-branches case is given in [27]. This formula depends on the first two Puiseux exponents (which are $\bar{\beta}_{0}$ and $\bar{\beta}_{1}$ ) of the branches and on its intersection number, and in many cases, the log-canonical threshold is given as the minimum of three candidates. Furthermore, in the same paper, it is also proved that for any number of branches, the log-canonical threshold only depends on the first two Puiseux exponents of the branches and their intersection numbers; however no formula is given. Finally, in $[4, \mathbf{3}]$, it has been proved that (even in the non-reduced case), there exist suitable local coordinates such that the log-canonical threshold coincides with the intersection of the Newton polygon of the curve with the diagonal line. 
In this paper, we give in Theorem 2.13 an explicit formula for the log-canonical threshold of a reduced (germ of) plane curve $C$. Our formula extends that of $[\mathbf{2 7}]$ to any number of branches. We also improve the formula of [27] determining the value of the log-canonical threshold for the two branches case without having to calculate a minimum (see Corollary 2.18). Since, in our case, there exists a log resolution in any characteristic and our methods do not depend on it, our formulae hold for any reduced curve in any characteristic. As a byproduct of our result we provide a formula for the log-canonical threshold of a non-reduced plane curve and also for a (non-necessarily simple) complete ideal of finite co-length in the power series ring $k[[x, y]]$ (see Remarks 2.19 and 2.20).

Next, we briefly describe the contents of the paper. Section 1 reviews some definitions and properties we need for stating and proving our results; moreover, we give an example of a curve with 8 branches whose minimal $\log$ resolution is represented by its dual graph, object that is an important tool in our development. Our example is used in the paper to explain notations and results. A simpler example with 3 branches is also provided for helping the reader to get a complete understanding of our ideas. In Section 2 we state our main result, Theorem 2.13, which is proved in Section 3. One step in the proof is Proposition 2.3, which asserts that to compute the $\log$-canonical threshold of $C$, we only need to take into account those divisors of the minimal $\log$ resolution $\pi$ of $C$ associated with the first two characteristic exponents [12, III.2] of each branch. For the complex case, this fact can be deduced either from $[\mathbf{2 7}]$ or $[\mathbf{1 8}]$. Our proof is valid for any characteristic, it is essentially algebraic and supported in some results from Delgado in [15]. Finally, we add that the formula in [27] for the two branches case is a consequence of Theorem 2.13 and an improvement of that formula is stated as Corollary 2.18 in Section 2.

Theorem 2.13, has two parts: The first one determines an exceptional divisor $E_{k}$ of $\pi$ which provides the log-canonical threshold. The second part uses this index $k$ to show the exact value of the log-canonical threshold in terms of the intersection multiplicities between branches and the first two maximal contact values of each branch [12, IV.2].

Being more specific for part one, we define a weight function (see $(2 \cdot 2))$ over those vertices $\mathbf{v}_{j}$ in the dual graph $\Gamma(C)$ of $\pi$ given by Proposition 2.3. This weight is easily computable as a difference that involves at most the first two maximal contact values of each branch. For getting the minuend and subtrahend, we delete $\mathbf{v}_{j}$ from $\Gamma(C)$ and distinguish among branches attached, or not, to the connected component containing the initial vertex $\mathbf{v}_{1}$. Set $\mathcal{V}$ the set of weighted vertices whose adapted degree (see Definition $2 \cdot 4$ ) is larger that two. Then, the first part shows that $E_{k}$ corresponds to the end vertex of a distinguished path in $\Gamma(C)$. This path is the only one joining $\mathbf{v}_{1}$ with a vertex in $\mathcal{V}$ such that the weights of their vertices in $\mathcal{V}$ are negative, while the ones of the remaining vertices in $\mathcal{V}$ are not.

In Section 3, we give the proofs of Proposition 2.3 and Theorem 2.13 by means of several auxiliary results. Lemmas $3 \cdot 1$ to $3 \cdot 7$ allow us to show Proposition 2.3 and they are also useful for the proof of Theorem $2 \cdot 13$. The first part of this theorem is proved with the help of three more lemmas which are uniquely based in combinatorics on the dual graph. The main step to show its second part is Lemma 3.12, where a comparison among candidates for log-canonical threshold is given. The proof of this lemma is supported on previous results of the paper and on a suitable choice of different partitions of the set of branches of the curve $C$. 


\section{Preliminaries}

Let $R=k[[x, y]]$ be the formal power series ring with coefficients over an algebraically closed field $k$. Consider a reduced series $f \in R$ and its decomposition $f=f_{1} f_{2} \cdots f_{r}$ as a product of irreducible elements $f_{i}$ in $R$. Denote by $C$ (respectively, $C_{i}, 1 \leqslant i \leqslant r$ ) the divisors on $\operatorname{Spec}(R)$ (that is, the germs of plane curves) defined by $f$ (respectively, $f_{i}$ ). Assume also that $C$ is singular and $r>2$ or $r=2$ but $C_{1}$ and $C_{2}$ are not non-singular and transversal. A $\log$ resolution of $C$ is a composition of finitely many blowing-ups centered at closed points

$$
\pi: X=X_{m} \stackrel{\pi_{m}}{\longrightarrow} X_{m-1} \longrightarrow \cdots \longrightarrow X_{1} \stackrel{\pi_{1}}{\longrightarrow} X_{0}=\operatorname{Spec}(R),
$$

$E_{j}$ being the exceptional divisor of $\pi_{j}, 1 \leqslant j \leqslant m$, such that $(f) \cdot \mathcal{O}_{X}=\mathcal{O}_{X}(-F)$, where $F$ is an effective divisor on $X$ which has simple normal crossing support. By an abuse of notation, the strict transform of $E_{j}$ on $X$ will also be denoted by $E_{j}$. From now on we will assume that $\pi$ is a minimal (with respect to the number of involved blowing-ups) $\log$ resolution of $C$. Notice that it exists and is unique (see [8], for instance).

According to the Introduction, if $C, \pi$ and $f$ are as above, we can associate with each positive rational number $t$ a multiplier ideal $\pi_{*} \mathcal{O}_{X}\left(K_{X \mid X_{0}}-\lfloor t F\rfloor\right)$ which we will denote by $\mathcal{J}\left(X_{0}, t C\right)$. The log-canonical threshold of $C$, $\operatorname{lct}(C)$, will be the smallest positive rational number $\iota_{1}$ such that $\mathcal{J}\left(X_{0}, \iota_{1} C\right) \neq R$.

Let $O$ be the closed point of $\operatorname{Spec}(R)$ and $\mathcal{C}=\left\{P_{1}=O, P_{2}, \ldots, P_{m}\right\}$ the set of closed points such that $P_{j}$ is the center of $\pi_{j}, 1 \leqslant j \leqslant m ; \mathcal{C}$ is a constellation of infinitely near points [14] and $\mathcal{C}$ can be written as a union of constellations $\mathcal{C}=\cup_{i=1}^{r} \mathcal{C}_{i}$ such that, for each $i=1, \ldots, r, \mathcal{C}_{i}$ is the constellation of points of $\mathcal{C}$ through which the successive strict transforms (by the blowing-ups in (1.1)) of the branch $C_{i}$ pass. Given two points $P_{j}, P_{k} \in \mathcal{C}$ with $k \geqslant j, P_{k}$ is called infinitely near to $P_{j}$ (and denoted $P_{k} \gtrsim P_{j}$ ) if the composition of blowing-ups $X_{k-1} \rightarrow X_{j-1}$ maps $P_{k}$ to $P_{j}$; notice that $\gtrsim$ is a partial ordering on $\mathcal{C}$. If, in addition, $P_{k} \neq P_{j}$ and $P_{k}$ belongs to the strict transform of $E_{j}$ on $X_{k-1}$, then $P_{k}$ is said to be proximate to $P_{j}$, which is denoted by $P_{k} \rightarrow P_{j}$. When $P_{k}$ is proximate to 2 points (respectively, 1 point) of $\mathcal{C}$ we name $P_{k}$ a satellite (respectively, free) point.

The first infinitesimal neighborhood of a point $P_{j} \in \mathcal{C}$ is the family of closed points belonging to the exceptional divisor obtained by the blowing-up at $P_{j}$ and the $l$ th infinitesimal neighborhood of $P_{j}(l>1)$ is (inductively defined) the set of points in the first infinitesimal neighborhood of some point in the $(l-1)$ th infinitesimal neighborhood of $P_{j}$.

It is well-known that $\pi^{*} C=\tilde{C}+\sum_{j=1}^{m} \mathbf{n}_{j} E_{j}^{*}$, where $E_{j}^{*}$ is the total transform of $E_{j}$ and $\tilde{C}$ the strict transform of $C$, both on $X, \mathbf{n}_{j}$ being the multiplicity of the strict transform of $C$ at $P_{j}, 1 \leqslant j \leqslant m$. We have also that

$$
E_{j}=E_{j}^{*}-\sum_{P_{k} \rightarrow P_{j}} E_{k}^{*} .
$$

Writing $\pi^{*} C=\tilde{C}+\sum_{j=1}^{m} b_{j} E_{j}$ and $K_{X / X_{0}}=\sum_{j=1}^{m} a_{j} E_{j}$ (the relative canonical divisor), the following equality holds (notice that $C$ is assumed to be singular):

$$
\operatorname{lct}(C)=\min \left\{\bar{\alpha}_{j}:=\frac{a_{j}+1}{b_{j}} \mid j=1,2, \ldots, m\right\} .
$$

In some occasions, the values $\bar{\alpha}_{j}$ will be named candidates for log-canonical thresh- 
old of $C$. The proximity matrix of $\mathcal{C}$ is the matrix $\mathbf{P}=\left(p_{k j}\right)_{k, j=1}^{m}$ defined by $p_{k k}=$ 1 for all $k, p_{k j}=-1$ if $P_{k} \rightarrow P_{j}$ and 0 otherwise. Notice that $\left(b_{1}, b_{2}, \ldots, b_{m}\right)^{t}:=$ $\mathbf{P}^{-1}\left(\mathbf{n}_{1}, \mathbf{n}_{2}, \ldots, \mathbf{n}_{m}\right)^{t}$ and $\left(a_{1}, a_{2}, \ldots, a_{m}\right)^{t}:=\mathbf{P}^{-1}(1,1, \ldots, 1)^{t}$.

The dual graph of $C, \Gamma(C)$, is an important object in our development. It is an oriented tree such that the strict transform (on $X$ ) of each exceptional divisor $E_{j}$ is represented by a vertex, $\mathbf{v}_{j}$. Two vertices are connected by an edge if the corresponding divisors meet. The strict transform (on $X$ ) $\tilde{C}_{i}$ of each component $C_{i}, i=1, \ldots, r$, is represented by an arrow, $\mathbf{a}_{i}$, which is a label of the vertex associated with the exceptional divisor whose strict transform meets $\tilde{C}_{i}$ (or, in other words, the maximum point of $\mathcal{C}_{i}$ for the ordering $\lesssim$ ). Usually each vertex $\mathbf{v}_{j}$ is labeled by the number $j$ (that is, the number of blowing-ups in the sequence (1·1) needed to create the corresponding divisor). The initial vertex of the edge that joins two vertices $\mathbf{v}_{j_{1}}$ and $\mathbf{v}_{j_{2}}$ is the one labeled with $\min \left\{j_{1}, j_{2}\right\}$.

We use the dual graph complemented with the so-called proximity (oriented) graph of $C$ for representing the minimal log resolution of $\pi$. The proximity graph allows us to decide which vertices are involved in the resolution of each branch. Its vertices correspond with the points in $\mathcal{C}$ and its edges join proximate points. An edge joining $P_{k}$ and $P_{j}(k>j)$ is a continuous straight line whenever $P_{k}$ is in the first infinitesimal neighborhood of $P_{j}$, otherwise it is a dotted curved line. By convention, we will omit those dotted curved edges which are deduced from others. As in the dual graph, we label with an arrow $\mathbf{a}_{i}$ the vertices corresponding with the maximum point in $\mathcal{C}_{i}$.

EXAMPLE $1 \cdot 1$

A. Figure 1 shows the proximity and dual graphs (where we have omitted the orientation) of a reduced curve $C$ with 8 components, $C_{1}, C_{2}, \ldots, C_{8}$, defined by 8 irreducible elements $f_{1}, f_{2}, \ldots, f_{8} \in R$, such that its minimal $\log$ resolution $\pi$ is obtained by blowingup a constellation, $\mathcal{C}=\left\{P_{j}\right\}_{j=1}^{17}$, of 17 infinitely near points. The reader can see additional labels in some vertices of the dual graph (thicker dots, stars and squares) that will be explained in forthcoming examples. One has that $\mathcal{C}=\cup_{i=1}^{8} \mathcal{C}_{i}$, where

$$
\begin{gathered}
\mathcal{C}_{1}=\left\{P_{1}, P_{2}, P_{3}, P_{4}, P_{5}, P_{6}, P_{7}\right\}, \mathcal{C}_{2}=\left\{P_{1}, P_{2}, P_{3}, P_{4}, P_{5}, P_{8}\right\}, \\
\mathcal{C}_{3}=\left\{P_{1}, P_{2}, P_{3}, P_{4}, P_{9}, P_{10}, P_{11}\right\}, \mathcal{C}_{4}=\left\{P_{1}, P_{2}, P_{3}, P_{4}, P_{9}, P_{10}, P_{12}, P_{13}\right\}, \\
\mathcal{C}_{5}=\left\{P_{1}, P_{2}, P_{16}, P_{17}\right\}, \quad \mathcal{C}_{6}=\mathcal{C}_{7}=\left\{P_{1}, P_{2}, P_{3}, P_{4}, P_{14}, P_{15}\right\} \\
\text { and } \mathcal{C}_{8}=\left\{P_{1}, P_{2}, P_{16}\right\} .
\end{gathered}
$$

Notice that, taking into account our mentioned convention, in the proximity graph we have omitted the dotted curved edge corresponding with the proximity $P_{5} \rightarrow P_{3}$ because it can be deduced from the proximity $P_{6} \rightarrow P_{3}$. The points $P_{1}, P_{2}, P_{3}, P_{4}, P_{9}, P_{10}, P_{12}, P_{14}$, $P_{15}$ and $P_{16}$ are free and the remaining ones satellite. In addition the curves $C_{6}, C_{7}$ and $C_{8}$ are nonsingular.

B. We also give a simpler example for a better understanding of our ideas. For simplicity, we use the same notation as in item A. So, we consider a reduced curve $C$ with three components $C_{i}$ defined by irreducible elements $f_{i} \in R, 1 \leqslant i \leqslant 3$, whose minimal $\log$ resolution is given by a constellation $\mathcal{C}=\left\{P_{j}\right\}_{j=1}^{20}$ with 20 infinitely near points. Proximity and dual graphs are depicted in Figure 2. 


\section{Results}

In this section we keep the notation given in the previous one. Let $h$ be an irreducible element in $R$ such that $\pi$ is a $\log$ resolution of the (germ of) curve $H$ that it defines. Let $\left\{\bar{\beta}_{l}^{h}\right\}_{l=0}^{g}$ be its maximal contact values (see Section 2 of Chapter IV in [12], for instance). This set of values is an increasing sequence of positive integers which is a minimal generating set of the so-called semigroup of values of $H$. In addition, they constitute an equivalent datum to the embedded topological type of $H$ and, in the complex case, can be easily computed from the set of Puiseux pairs given by a primitive parametrization of the curve. In this case $g$ is the number of Puiseux pairs and, in the general case, $g-1$ coincides with the number of vertices of degree 3 in the dual graph of $H$. Fundamentally we will use the values $\bar{\beta}_{0}^{h}$ and $\bar{\beta}_{1}^{h}$. The value $\bar{\beta}_{0}^{h}$ (respectively, $\bar{\beta}_{1}^{h}$ ) can be computed as the intersection multiplicity between $H$ and a (germ of) transversal line at the singular point of $H$ (respectively, a (germ of) curve sharing with $H$ all free points $P_{1}, \ldots, P_{\ell}$ in $\mathcal{C}$ before the first satellite point and such that its strict transform is regular and transversal to the exceptional divisor created by blowing-up at $P_{\ell}$, at a nonsingular point of the exceptional locus). When $H$ is nonsingular, the unique defined maximal contact value is $\bar{\beta}_{0}^{h}=1$. In this paper and in this case, we also define $\bar{\beta}_{1}^{h}$ as the number of points of $\mathcal{C}$ through which the strict transforms of $H$ pass.

Definition 2.1. Let $h$ and $H$ be as above. A terminal satellite point for $h$ (or for $H$ )
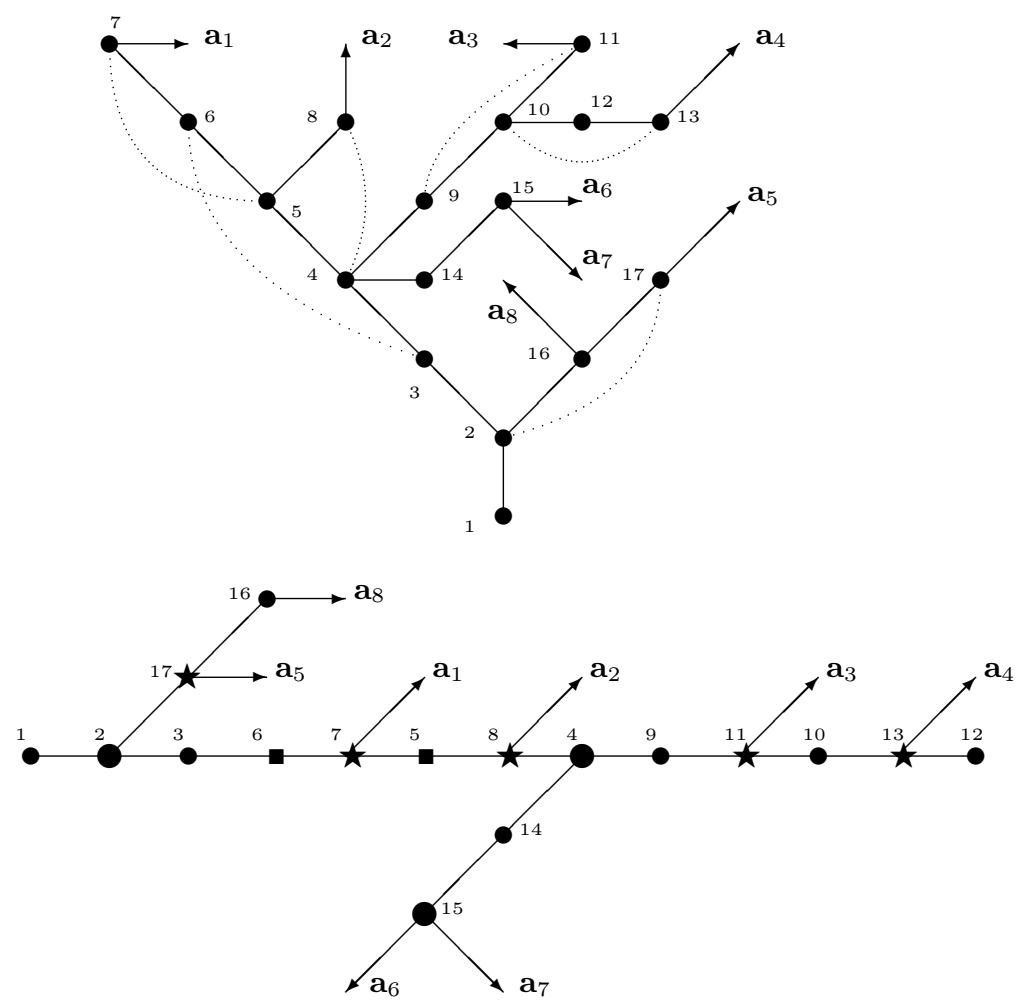

Fig. 1. Proximity and dual graphs of Example 1·1.A. 

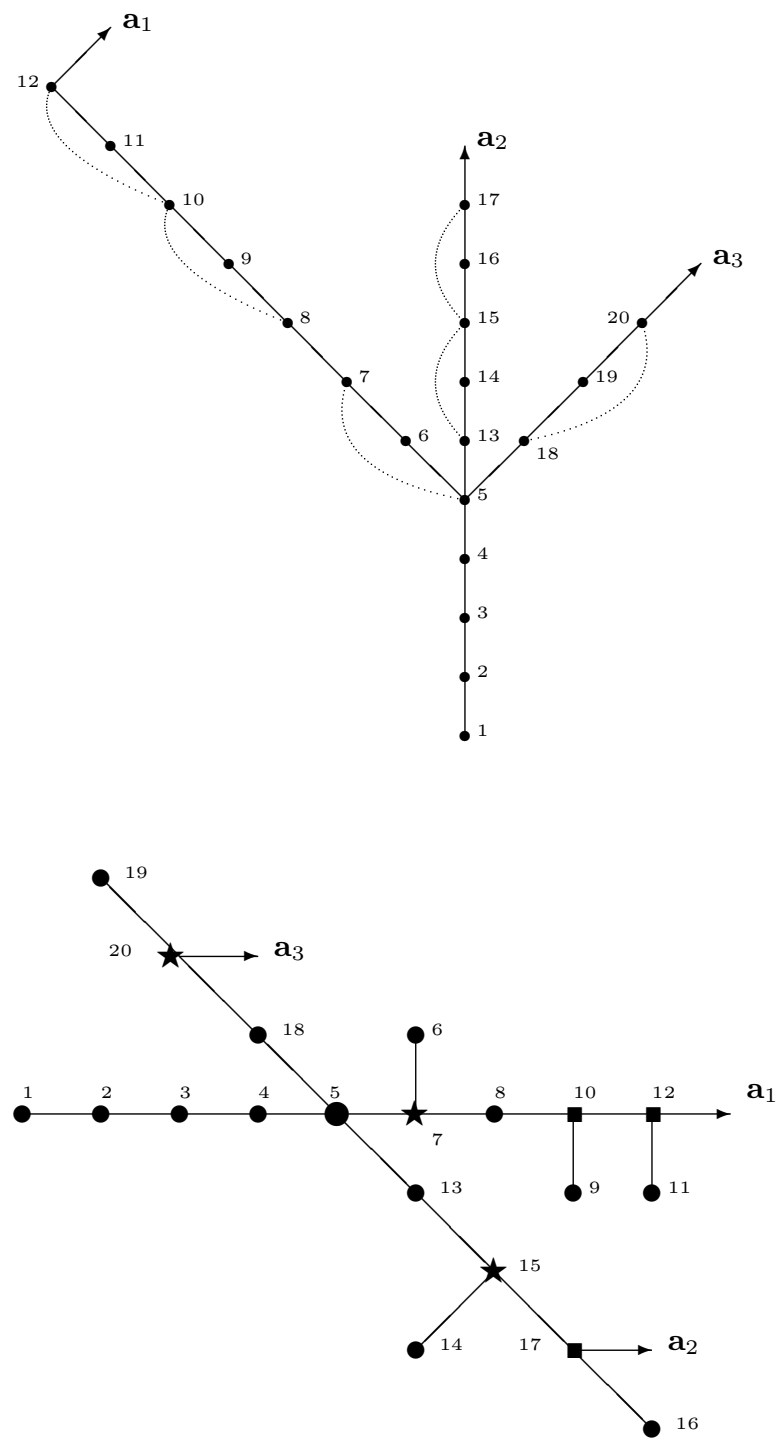

Fig. 2. Proximity and dual graphs of Example 1·1.B.

is a point $P_{j} \in \mathcal{C}$ such that it is satellite and the set

$\left\{P_{k} \in \mathcal{C} \backslash\left\{P_{j}\right\} \mid\right.$ the strict transform of $H$ on $X_{k}$ passes through $P_{k}$ and $\left.P_{k} \gtrsim P_{j}\right\}$ is either empty or its minimum (with respect to the ordering $\gtrsim$ ) is a free point.

Notice that the number of terminal satellite points for $h$ coincides with the index $g$ of the last maximal contact value $\bar{\beta}_{g}^{h}$ and that the part of the dual graph of $H$ up to the $l$ th terminal satellite point, $1 \leqslant l \leqslant g$, can be deduced from the set of values $\left\{\bar{\beta}_{0}^{h}, \bar{\beta}_{1}^{h}, \ldots, \bar{\beta}_{l}^{h}\right\}$. When the curve $H$ is not singular, we denote by $l_{0}^{h}$ the cardinality of the set of free points $P_{j} \in \mathcal{C}$ through which the strict transforms of $H$ pass. Otherwise, $l_{0}^{h}+1$ will stand for the 
cardinality of the set of free points $P_{j} \in \mathcal{C}$ satisfying the above condition and such that every terminal satellite point for $h$ is infinitely near to $P_{j}$ (see [15] for the source of the notation); in other words, $l_{0}^{h}+1$ is the length $\ell$ of the maximal chain of initial consecutive free points $P_{1} \lesssim \ldots \lesssim P_{\ell}$ such that the strict transforms of $H$ pass through $P_{i}$ for all $i \in\{1, \ldots, \ell\}$. Note that $\bar{\beta}_{0}^{h}$ is the multiplicity of the curve $H$ and $\bar{\beta}_{1}^{h}$ the intersection multiplicity between $H$ and a nonsingular curve passing through $P_{1}, P_{2}, \ldots, P_{\ell}$. When $h=f_{i}$ for some $i \in\{1,2, \ldots, r\}$, we have set $C_{i}$ instead of $F_{i}$ and, for simplicity, we will write $\bar{\beta}_{k}^{i}$ (respectively, $l_{0}^{i}$ ) instead of $\bar{\beta}_{k}^{f_{i}}$ (respectively, $l_{0}^{f_{i}}$ ).

Assume from now on that the curves $C_{1}, \ldots, C_{n}$ are singular and the remaining ones, $C_{n+1}, \ldots, C_{r}$, are nonsingular. For each $i \in\{1,2, \ldots, n\}$, we denote by $P_{t_{i}}$ the minimum terminal satellite point for $C_{i}$ with respect to the ordering $\lesssim$. The following two sets will be useful:

$$
\mathcal{T}:=\left\{P_{t_{i}} \mid i=1, \ldots, n\right\}
$$

and

$$
\mathcal{F}:=\left\{P_{j} \in \mathcal{C} \mid P_{j} \lesssim P_{t_{i}} \text { for some } P_{t_{i}} \in \mathcal{T}\right\} \cup \mathcal{C}_{n+1} \cdots \cup \mathcal{C}_{r} .
$$

Let us consider the above given examples with the aim of clarifying previous concepts.

Example 2.2. Consider again the curve in Example 1.1.A. Then $n=5$ and, from the proximity graph, one can see that

$$
\mathcal{T}=\left\{P_{t_{1}}=P_{7}, P_{t_{2}}=P_{8}, P_{t_{3}}=P_{11}, P_{t_{4}}=P_{13}, P_{t_{5}}=P_{17}\right\} .
$$

In the dual graph depicted in Figure 1, we have labeled those vertices representing divisors $E_{j}$ such that $P_{j}$ is a satellite point and it is not in $\mathcal{T}$ (respectively, it is in $\mathcal{T}$ ) with a square (respectively, a star). Notice that, in this example, $\mathcal{F}=\mathcal{C}$.

The free points of $\mathcal{C}_{1}$ such that the unique terminal satellite point in $\mathcal{C}_{1}$ is proximate to them (that is, the initial free points in the branch of the proximity graph corresponding to $\mathcal{C}_{1}$ ) are $P_{1}, P_{2}, P_{3}$ and $P_{4}$. Therefore $l_{0}^{1}+1=4$. Similarly $l_{0}^{2}=3, l_{0}^{3}=5, l_{0}^{4}=6, l_{0}^{5}=$ $2, l_{0}^{6}=l_{0}^{7}=6$ and $l_{0}^{8}=3$.

With respect to the curve in Example 1.1.B, one gets $n=3, \mathcal{T}=\left\{P_{t_{1}}=P_{7}, P_{t_{2}}=\right.$ $\left.P_{15}, P_{t_{3}}=P_{20}\right\}$ and

$$
\mathcal{F}=\mathcal{C} \backslash\left\{P_{8}, P_{9}, P_{10}, P_{11}, P_{12}, P_{16}, P_{17}\right\} .
$$

Next, we will state our first result which, in the complex case, can be deduced from the proof of [27, Theorem 1.3]. This proof uses results in [26] developed for complex numbers. Our result shows that the $\log$-canonical threshold of $C$ depends only on the part of the dual graph determined by the values $\bar{\beta}_{0}^{i}$ and $\bar{\beta}_{1}^{i}$ of every component $C_{i}$ of $C$.

Proposition 2.3. With the notation given before Equality (1.2), it holds that the log-canonical threshold of $C$ is the minimum of the elements in the set

$$
\left\{\bar{\alpha}_{j}=\frac{a_{j}+1}{b_{j}} \mid P_{j} \in \mathcal{F}\right\} .
$$

Our main result uses a weight function for some vertices of $\Gamma(C)$. Before stating it, we introduce these weights and some other necessary concepts.

Recall that $C$ is a reduced curve with $r$ branches and let $\mathcal{V}_{\mathcal{F}}$ be the set of vertices $\mathbf{v}_{j}$ of $\Gamma(C)$ such that $P_{j} \in \mathcal{F}$. 
Definition 2.4. The adapted degree of a vertex $\mathbf{v}_{j} \neq \mathbf{v}_{1}$ (respectively, $\mathbf{v}_{1}$ ) of the dual graph $\Gamma(C)$ is defined as the sum of its degree and the number of arrows labeling it (respectively, one plus the number we have just defined).

The set of vertices in $\mathcal{V}_{\mathcal{F}}$ with adapted degree greater than or equal to 3 will be denoted by $\mathcal{V}$.

Set $\mathcal{S}$ the set of free points in the configuration $\mathcal{C}$ corresponding with vertices in $\mathcal{V}$. Clearly, $\mathcal{T}$ is that of satellite ones. Then, the following notations will be used along the paper

$$
\begin{gathered}
\mathcal{V}_{\mathcal{T}}:=\left\{\mathbf{v}_{j} \mid P_{j} \in \mathcal{T}\right\}, \quad \mathcal{V}_{\mathcal{S}}:=\left\{\mathbf{v}_{j} \mid P_{j} \in \mathcal{S}\right\}, \quad \mathcal{V}_{\text {free }}:=\left\{\mathbf{v}_{j} \in \mathcal{V}_{\mathcal{F}} \mid P_{j} \text { is a free point }\right\}, \\
\mathcal{V}_{\text {end }}:=\left\{\mathbf{v}_{j} \in \mathcal{V}_{\mathcal{F}} \mid \mathbf{v}_{j} \text { has degree } 1\right\} \quad \text { and obviously } \mathcal{V}=\mathcal{V}_{\mathcal{T}} \cup \mathcal{V}_{\mathcal{S}} .
\end{gathered}
$$

$\mathcal{V}_{\text {end }}$ is a subset of the set formed with the vertex $\mathbf{v}_{1}$ and those vertices $\mathbf{v}_{j}$ such that $P_{j}$ is a maximal free point of $\mathcal{F}$ (with respect to the ordering $\lesssim$ ).

Example 2.5. Consider again Example 1·1.A. Then, $\mathcal{S}=\left\{P_{2}, P_{4}, P_{15}\right\}$. These points have been marked with thicker dots in the dual graph depicted in Figure 1. In Figure 2, we have used the same mark for the dual graph of the curve in Example 1.1.B and, here, $\mathcal{S}$ is reduced to one point, $\mathcal{S}=\left\{P_{5}\right\}$.

As usual, a path $\gamma$ in $\Gamma(C)$ is an ordered sequence of different vertices of $\Gamma(C)$ such that two consecutive ones are joined by an (oriented) edge. If $a$ is the initial (or first) vertex, in $(\gamma$ ), and $b$ the terminal (or last) vertex (which can also be given by an arrow, if any, that labels it), $\operatorname{ter}(\gamma)$, we will denote $\gamma$ by $[a, b]$ and also, by an abuse of notation, its set of vertices. Moreover, we will use $] a, b]$ (respectively, $] a, b[$ ) to denote the path (or its set of vertices) obtained after removing from $\gamma$ the initial vertex (respectively, the initial and terminal vertices) and its incident edge (respectively, incident edges).

Let $\leqslant$ denote the order induced by the (oriented) tree $\Gamma(C)$ in its set of vertices, that is, given two vertices $\mathbf{v}_{j_{1}}$ and $\mathbf{v}_{j_{2}}, \mathbf{v}_{j_{1}} \leqslant \mathbf{v}_{j_{2}}$ means that $\mathbf{v}_{j_{1}}$ belongs to $\left[\mathbf{v}_{1}, \mathbf{v}_{j_{2}}\right]$. By convention, if $\mathbf{a}_{i}$ is an arrow that is a label of $\mathbf{v}_{j_{2}}$ then $\mathbf{v}_{j_{1}} \leqslant \mathbf{a}_{i}$ will mean $\mathbf{v}_{j_{1}} \leqslant \mathbf{v}_{j_{2}}$.

Also, for every vertex $\mathbf{v}_{j} \in \mathcal{V}_{\mathcal{F}}$, we define

$$
\mathbf{v}_{j}^{<}:=\left\{\mathbf{a}_{i} \mid \mathbf{v}_{j} \not \leq \mathbf{a}_{i}\right\} \text { and } \mathbf{v}_{j}^{\geqslant}:=\left\{\mathbf{a}_{i} \mid \mathbf{v}_{j} \leqslant \mathbf{a}_{i}\right\} .
$$

Furthermore we consider the map $\sigma: \mathcal{V}_{\mathcal{F}} \rightarrow \mathbb{Z}$ given by

$$
\sigma\left(\mathbf{v}_{j}\right)=\sum_{\mathbf{a}_{i} \in \mathbf{v}_{j}^{<}} c_{j i} \bar{\beta}_{0}^{i}-\sum_{\mathbf{a}_{i} \in \mathbf{v}_{j}^{\geqslant}} \bar{\beta}_{0}^{i},
$$

where

$$
c_{j i}:= \begin{cases}\operatorname{card}\left(\left[\mathbf{v}_{1}, \mathbf{a}_{i}\right] \cap\left[\mathbf{v}_{1}, \mathbf{v}_{j}\right] \cap \mathcal{V}_{\text {free }}\right) & \text { if } \operatorname{ter}\left(\left[\mathbf{v}_{1}, \mathbf{a}_{i}\right] \cap\left[\mathbf{v}_{1}, \mathbf{v}_{j}\right]\right) \in \mathcal{V}_{\mathcal{S}}, \\ \bar{\beta}_{1}^{i} / \bar{\beta}_{0}^{i} & \text { otherwise. }\end{cases}
$$

With these data, we are able to state Part (1) and the first assertion in Part (2) of Theorem 2.13, which show a vertex $\mathbf{v}_{k}$ of the dual graph $\Gamma(C)$ providing the log-canonical threshold $\bar{\alpha}_{k}$ of $C$. Next, we introduce the above mentioned necessary concepts that will be useful in the next section and to state Part (2) of Theorem 2.13.

Definition 2.6. Let $h_{1}$ and $h_{2}$ be two irreducible elements in $R$ such that $\pi$ is a 
common log resolution of the curves $H_{1}$ and $H_{2}\left(\neq H_{1}\right)$ that they define. The contact pair of $h_{1}$ and $h_{2},\left(h_{1} \mid h_{2}\right)$, is defined to be the couple of integers $(q, c)$ such that:

- $q$ is the number of common terminal satellite points for $h_{1}$ and $h_{2}$.

- $c$ is the cardinality of the set of free points $P_{j} \in \mathcal{C}$ such that the strict transforms of $H_{1}$ and $H_{2}$ pass through $P_{j}$ and $P_{j}$ is infinitely near to the last common terminal satellite point (if any).

An equivalent definition of contact pair using the Hamburger-Noether expansion of the branches is given in [15].

ExAmple $2 \cdot 7$. Let us return to Example 1·1.A. $C_{1}$ and $C_{3}$ do not share any terminal satellite point and, moreover, the free points in $\mathcal{C}_{1} \cap \mathcal{C}_{3}$ are $P_{1}, P_{2}, P_{3}$ and $P_{4}$; therefore $\left(f_{1} \mid f_{3}\right)=(0,4)$.

Now, suppose that $h_{1}$ and $h_{2}$ are irreducible elements in $R$ such that the minimal $\log$ resolution of the curve defined by $h_{1}$ (respectively, $h_{2}$ ) is given by blowing-up at the points $P_{1}, P_{2}, P_{16}, P_{17}$ and $P_{18}, P_{19}$ (respectively, and $\left.P_{20}, P_{21}\right) . P_{18}, P_{19}, P_{20}, P_{21}$ are new points enlarging $\mathcal{C}$ up to a new configuration $\mathcal{C}^{\prime}$ such that all of them are proximate to $P_{17}, P_{18}$ and $P_{20}$ are two distinct points in the first infinitesimal neighborhood of $P_{17}$, $P_{19} \gtrsim P_{18}$ and $P_{21} \gtrsim P_{20}$. Set also $\pi$ the $\log$ resolution given by $\mathcal{C}^{\prime}$. Then, each curve $H_{1}$ and $H_{2}$ has two terminal satellite points and $\left(h_{1} \mid h_{2}\right)=(1,0)$ because $P_{17}$ is their last common infinitely near point.

Definition 2.8. Two components $C_{i_{1}}$ and $C_{i_{2}}$ of the curve $C$ are called to be separated at a point $P_{j} \in \mathcal{C}$ when $\max _{\gtrsim}\left(\mathcal{C}_{i_{1}} \cap \mathcal{C}_{i_{2}}\right)=P_{j}$.

Moreover, $C_{i_{1}}$ and $C_{i_{2}}$ will be freely separated at $P_{j}$ if they are separated at $P_{j}$ and $\left(f_{i_{1}} \mid f_{i_{2}}\right)=(0, c)$ for some $c \leqslant \min \left\{l_{0}^{i_{1}}, l_{0}^{i_{2}}\right\}$.

Finally, a point $P_{j} \in \mathcal{C}$ is an initial separating point if at least two components $C_{i_{1}}$ and $C_{i_{2}}$ of $C$ are freely separated at $P_{j}$.

Remark 2.9. Notice that if two components of $C$ are freely separated at a point $P_{j}$, then $P_{j}$ must be a free point. As a consequence, $\mathcal{S}=\{$ initial separating points of $\mathcal{C}\}$.

The following definition will allow us to clarify the meaning of the coefficients $c_{i j}$ and the condition ter $\left(\left[\mathbf{v}_{1}, \mathbf{a}_{i}\right] \cap\left[\mathbf{v}_{1}, \mathbf{v}_{j}\right]\right) \in \mathcal{V}_{\mathcal{S}}$ appearing in the definition of our map $\sigma$ (see Remark 2.11).

Definition 2.10. Consider a point $P_{j} \in \mathcal{C}, \mathcal{C}$ being the configuration of the mentioned resolution $\pi$ of $C$. A curvette at $P_{j}$ will be an irreducible element $\varphi \in R$ defining a divisor on $\operatorname{Spec}(R)$ different from $C_{i}$ for all $i \in\{1,2, \ldots, r\}$, whose strict transform on $X$ is not singular and meets $E_{j}$ transversally at a regular point.

REMARK 2.11. Notice that, if $\mathbf{v}_{j} \in \mathcal{V}_{\mathcal{F}}$ and $\mathbf{a}_{i} \in \mathbf{v}_{j}^{<}$, then the condition

$$
\operatorname{ter}\left(\left[\mathbf{v}_{1}, \mathbf{a}_{i}\right] \cap\left[\mathbf{v}_{1}, \mathbf{v}_{j}\right]\right) \in \mathcal{V}_{\mathcal{S}}
$$

is equivalent to say that a curvette at $P_{j}, \psi$, and $C_{i}$ are freely separated (as components of a reduced curve that contains both branches). Moreover, in this case, the integer $c_{j i}$ appearing in the definition of the map $\sigma$ coincides with the integer $c$ such that $\left(\psi \mid f_{i}\right)=$ $(0, c)$. 
REMARK 2.12. The sets $\mathbf{v}_{j}^{<}$and $\mathbf{v}_{j}^{\geqslant}$do not change when $\mathbf{v}_{j}$ runs over a path $] \mathbf{v}_{j_{1}}, \mathbf{v}_{j_{2}}$ ] such that $\mathbf{v}_{j_{1}}, \mathbf{v}_{j_{2}} \in \mathcal{V} \cup \mathcal{V}_{\text {end }}$ and $] \mathbf{v}_{j_{1}}, \mathbf{v}_{j_{2}}[\cap \mathcal{V}=\emptyset$. Therefore the map $\sigma$ is constant in such a path.

Let us denote by $I\left(f_{i}, f_{s}\right)$ the intersection multiplicity of two different components $C_{i}$ and $C_{s}, 0 \leqslant i, s \leqslant r$, of $C$. We are ready to state our main result, which is:

TheOREM 2.13. Let $C$ be a singular reduced (germ of) plane curve with $r$ branches and $\mathcal{C}$ (respectively, $\Gamma(C)$ ) the constellation of infinitely near points (respectively, dual graph) associated with its minimal log resolution. Consider the subsets $\mathcal{T}$ and $\mathcal{S}$ of $\mathcal{C}$ and the subset of vertices $\mathcal{V}=\mathcal{V}_{\mathcal{T}} \cup \mathcal{V}_{\mathcal{S}}$ of $\Gamma(C)$ above defined. Then:

(1) There exists a vertex $\mathbf{v}_{k} \in \mathcal{V}$ satisfying the conditions:

(a) $\sigma\left(\mathbf{v}_{j}\right)<0$ for all $\mathbf{v}_{j} \in\left[\mathbf{v}_{1}, \mathbf{v}_{k}\right] \cap \mathcal{V}$ and

(b) $\sigma\left(\mathbf{v}_{j}\right) \geqslant 0$ for all $\mathbf{v}_{j} \in \mathcal{V} \backslash\left[\mathbf{v}_{1}, \mathbf{v}_{k}\right]$.

(2) The log-canonical threshold of $C$ is the value $\bar{\alpha}_{k}$ defined in the above equality (1.2) and it can be computed as follows:

- If $\mathbf{v}_{k}=\mathbf{v}_{t_{i}} \in \mathcal{V}_{\mathcal{T}}$, then

$$
\bar{\alpha}_{k}=\bar{\alpha}_{t_{i}}=\frac{\bar{\beta}_{0}^{i}+\bar{\beta}_{1}^{i}}{\sum_{s=1}^{r} \delta_{i s}}
$$

where

$$
\delta_{i s}=\left\{\begin{array}{l}
\bar{\beta}_{0}^{i} \bar{\beta}_{1}^{s} \quad \text { if either } s=i, \text { or } s \neq i \text { and } \bar{\beta}_{0}^{i} \bar{\beta}_{1}^{s}=\bar{\beta}_{1}^{i} \bar{\beta}_{0}^{s} \leqslant I\left(f_{i}, f_{s}\right), \\
I\left(f_{i}, f_{s}\right) \text { otherwise. }
\end{array}\right.
$$

- If $\mathbf{v}_{k} \in \mathcal{V}_{\mathcal{S}}$, then

$$
\bar{\alpha}_{k}=\frac{\bar{\beta}_{0}^{i_{1}} \bar{\beta}_{0}^{i_{2}}+I\left(f_{i_{1}}, f_{i_{2}}\right)}{\bar{\beta}_{0}^{i_{1}} I\left(f_{i_{1}}, f_{i_{2}}\right)+\bar{\beta}_{0}^{i_{2}} \sum_{1 \leqslant s \leqslant r, s \neq i_{1}} I\left(f_{i_{1}}, f_{s}\right)},
$$

where $C_{i_{1}}$ and $C_{i_{2}}$ are any two components which are freely separated at $P_{k}$.

Remark $2 \cdot 14$. Notice that the vertex $\mathbf{v}_{k}$ mentioned in Theorem 2.13 can be easily obtained applying, to the graph $\Gamma(C)$, an obvious variant of the well-known breadth-first search strategy used in graph theory.

EXAmple 2.15. Consider the curve in Example 1.1.A. The sets of maximal contact values of the components $C_{1}, C_{2}, \ldots, C_{5}$ are, respectively, $\left\{\bar{\beta}_{0}^{1}, \bar{\beta}_{1}^{1}\right\}=\{5,17\},\left\{\bar{\beta}_{0}^{2}, \bar{\beta}_{1}^{2}\right\}=$ $\{3,11\},\left\{\bar{\beta}_{0}^{3}, \bar{\beta}_{1}^{3}\right\}=\{2,11\},\left\{\bar{\beta}_{0}^{4}, \bar{\beta}_{1}^{4}\right\}=\{2,13\}$ and $\left\{\bar{\beta}_{0}^{5}, \bar{\beta}_{1}^{5}\right\}=\{2,5\}$. We have that

$$
\mathcal{V}=\mathcal{V}_{\mathcal{S}} \cup \mathcal{V}_{\mathcal{T}}=\left\{\mathbf{v}_{2}, \mathbf{v}_{4}, \mathbf{v}_{7}, \mathbf{v}_{8}, \mathbf{v}_{11}, \mathbf{v}_{13}, \mathbf{v}_{15}, \mathbf{v}_{17}\right\}
$$

Moreover

$$
\begin{gathered}
\sigma\left(\mathbf{v}_{2}\right)=-\sum_{i=1}^{8} \bar{\beta}_{0}^{i}=-17, \quad \sigma\left(\mathbf{v}_{7}\right)=2 \bar{\beta}_{0}^{5}+2 \bar{\beta}_{0}^{8}-\bar{\beta}_{0}^{1}-\bar{\beta}_{0}^{2}-\bar{\beta}_{0}^{3}-\bar{\beta}_{0}^{4}-\bar{\beta}_{0}^{6}-\bar{\beta}_{0}^{7}=-8 \\
\quad \text { and } \sigma\left(\mathbf{v}_{8}\right)=2 \bar{\beta}_{0}^{5}+2 \bar{\beta}_{0}^{8}+\bar{\beta}_{1}^{1}-\bar{\beta}_{0}^{2}-\bar{\beta}_{0}^{3}-\bar{\beta}_{0}^{4}-\bar{\beta}_{0}^{6}-\bar{\beta}_{0}^{7}=14 .
\end{gathered}
$$

Therefore, $\mathbf{v}_{7}$ is the distinguished vertex $\mathbf{v}_{k}$ in Theorem $2 \cdot 13$. Then

$$
\operatorname{lct}(C)=\bar{\alpha}_{7}=\bar{\alpha}_{t_{1}}=\frac{\bar{\beta}_{1}^{1}+\bar{\beta}_{0}^{1}}{\bar{\beta}_{1}^{1} \bar{\beta}_{0}^{1}+\sum_{s=2}^{8} I\left(f_{1}, f_{s}\right)}=
$$


With respect to the curve in Example 1.1.B, it holds: $\left\{\bar{\beta}_{0}^{1}, \bar{\beta}_{1}^{1}\right\}=\{8,44\},\left\{\bar{\beta}_{0}^{2}, \bar{\beta}_{1}^{2}\right\}=$ $\{4,26\},\left\{\bar{\beta}_{0}^{3}, \bar{\beta}_{1}^{3}\right\}=\{2,13\}$. Now, $\mathcal{V}=\left\{\mathbf{v}_{5}, \mathbf{v}_{7}, \mathbf{v}_{15}, \mathbf{v}_{20}\right\}$. Furthermore, $\sigma\left(\mathbf{v}_{5}\right)=-8-$ $4-2=-14, \sigma\left(\mathbf{v}_{7}\right)=5 \bar{\beta}_{0}^{2}+5 \bar{\beta}_{0}^{3}-\bar{\beta}_{0}^{1}=22, \sigma\left(\mathbf{v}_{15}\right)=5 \bar{\beta}_{0}^{1}+5 \bar{\beta}_{0}^{3}-\bar{\beta}_{0}^{2}=46$ and $\sigma\left(\mathbf{v}_{20}\right)=5 \bar{\beta}_{0}^{1}+5 \bar{\beta}_{0}^{2}-\bar{\beta}_{0}^{3}=58$. As a consequence, the distinguished vertex is $\mathbf{v}_{5}$ and

$$
\operatorname{lct}(C)=\bar{\alpha}_{5}=\frac{\bar{\beta}_{0}^{2} \bar{\beta}_{0}^{3}+I\left(f_{2}, f_{3}\right)}{\bar{\beta}_{0}^{2} I\left(f_{2}, f_{3}\right)+\bar{\beta}_{0}^{3}\left[I\left(f_{2}, f_{1}\right)+I\left(f_{2}, f_{3}\right)\right]}=\frac{3}{35} .
$$

REMARK 2.16. Notice that our Example 1·1.A might induce the reader to think that one has to consider a large number of vertices of $\Gamma(C)$. However, generally speaking, this is not true since we only need those vertices in $\mathcal{V} \subseteq \mathcal{V}_{\mathcal{F}}$, this last set being (proportionally) very small when $C$ has branches with many contact maximal values. We have considered a case in item A of Example 1.1 where rather vertices are useful to illustrate a wide spectrum of possibilities but avoiding unnecessary information. Our example in item B shows a more generic case.

REMARK $2 \cdot 17$. When the ground field $k$ is the field of complex numbers, it is proved in [34] that an exceptional divisor $E_{j}$ contributes some jumping number of $C$ if, and only if, $E_{j} \cdot\left(\left(\pi^{*} C\right)^{o}-E_{j}\right) \geqslant 3$, where $\left(\pi^{*} C\right)^{o}$ denotes the reduced divisor associated to $\pi^{*} C$. This fact and Proposition $2 \cdot 3$ prove that, in this case, the log-canonical threshold of $C$ is the minimum of the quotients $\bar{\alpha}_{j}$, where $\mathbf{v}_{j} \in \mathcal{V}_{\mathcal{F}}$ and the degree of $\mathbf{v}_{j}$ is greater than or equal to 3 . To define $\mathcal{V}$, we have used the concept of adapted degree instead of degree. Hence $\mathcal{V}$ could contain an additional vertex $\mathbf{v}_{1}$ of degree 2 . We have preferred to use this concept because it assures the uniqueness of the vertex $\mathbf{v}_{k}$ in the statement of Theorem $2 \cdot 13$ and simplifies the proofs. For instance, if $C=C_{1}+C_{2}$, where $C_{1}$ and $C_{2}$ are two unibranched equisingular (germs of) curves meeting transversally at $O=P_{1}$, then $\mathcal{V}$ has three vertices, including $\mathbf{v}_{1}$, and the values $\bar{\alpha}_{j}$ corresponding with those three vertices coincide.

As a consequence of Theorem $2 \cdot 13$ and the forthcoming Lemma $3 \cdot 1$, we state the following result which determines the exact value of the log-canonical threshold of a reduced curve with two branches.

Corollary 2.18. Assume that the number of components of $C$ is $r=2$ and, without loss of generality, that $\bar{\beta}_{1}^{1} / \bar{\beta}_{0}^{1} \leqslant \bar{\beta}_{1}^{2} / \bar{\beta}_{0}^{2}$. Then:

(a) If $C_{1}$ and $C_{2}$ are not freely separated, it holds that

$$
\operatorname{lct}(C)=\left\{\begin{array}{l}
\frac{\bar{\beta}_{1}^{1}+\bar{\beta}_{0}^{1}}{\bar{\beta}_{1}^{1}\left(\bar{\beta}_{0}^{1}+\bar{\beta}_{0}^{2}\right)} \text { if } \bar{\beta}_{1}^{1} \geqslant \bar{\beta}_{0}^{2}, \\
\frac{\bar{\beta}_{1}^{2}+\bar{\beta}_{0}^{2}}{\beta_{0}^{2}\left(\beta_{1}^{1}+\beta_{1}^{2}\right)} \text { otherwise. }
\end{array}\right.
$$

(b) If, on the contrary, $C_{1}$ and $C_{2}$ are freely separated,

$$
\operatorname{lct}(C)= \begin{cases}\frac{\bar{\beta}_{0}^{1} \bar{\beta}_{0}^{2}+I\left(f_{1}, f_{2}\right)}{\left(\bar{\beta}_{0}^{1}+\bar{\beta}_{1}^{2}\right) I\left(f_{1}, f_{2}\right)} & \text { if } \frac{1}{c} \leqslant \frac{\bar{\beta}_{0}^{2}}{\bar{\beta}_{0}^{1}} \leqslant c, \\ \frac{\bar{\beta}_{1}^{1} \bar{\beta}_{0}^{1}}{\bar{\beta}_{0}^{1} \bar{\beta}_{1}^{1}+I\left(f_{1}, f_{2}\right)} & \text { if } \frac{\bar{\beta}_{0}^{2}}{\bar{\beta}_{0}^{1}}<\frac{1}{c}, \\ \overline{\beta_{0}^{2}}, \bar{\beta}_{0}^{2} \bar{\beta}_{1}^{2}+\bar{\beta}_{1}^{2}+I\left(f_{1}, f_{2}\right) & \text { otherwise, }\end{cases}
$$


$c$ being the integer such that $\left(f_{1} \mid f_{2}\right)=(0, c)$.

We finish this section with two remarks concerning the log-canonical threshold of a non-reduced plane curve and of a complete ideal of our ring $R$.

Remark 2.19. Assume that $C=\sum_{i=1}^{r} n_{i} C_{i}$ is a non-reduced curve, $C_{1}, C_{2}, \ldots, C_{r}$ being its integral components. Let $\mathcal{C}$ be the configuration of infinitely near points associated with a $\log$-resolution of $C$. For each $i \in\{1,2, \ldots, r\}$, pick $n_{i}$ curves $C_{i 1}, C_{i 2}, \ldots, C_{i n_{i}}$ defined by $n_{i}$ curvettes at the maximal point $P_{j-1}$ of $\mathcal{C}$ through which the strict transform of $C_{i}$ passes and such that their strict transforms meet $E_{j}$ at different free points. Then, it follows from the definition of log-canonical threshold that

$$
\operatorname{lct}(C)=\min \left\{1 / n_{1}, 1 / n_{2}, \ldots, 1 / n_{r}, \operatorname{lct}\left(C^{\prime}\right)\right\},
$$

where $C^{\prime}$ is the reduced curve $\sum_{1 \leqslant i \leqslant r} \sum_{1 \leqslant j \leqslant n_{i}} C_{i j}$. Therefore Theorem $2 \cdot 13$ provides, in fact, a formula for the log-canonical threshold of any (reduced or non-reduced) singular plane curve.

REMARK $2 \cdot 20$. Theorem $2 \cdot 13$ also provides, as a byproduct, a formula for the logcanonical threshold of a complete ideal of finite co-length in $R$. Indeed, if $\mathfrak{a}$ is such an ideal, it has a unique factorization $\mathfrak{a}=\mathfrak{p}_{1}^{n_{1}} \cdots \mathfrak{p}_{r}^{n_{r}}$ as a product of simple complete ideals [41, page 385]. Then, it is straightforward from the definition that $\operatorname{lct}(\mathfrak{a})=\operatorname{lct}\left(\sum_{i=1}^{r} D_{i}\right)$ where, for each $i=1, \ldots, r, D_{i}$ is a sum of $n_{i}$ suitable chosen general curves of the ideal $\mathfrak{p}_{i}$. Recall that a general curve of a simple complete ideal $\mathfrak{p}_{i}$ is an irreducible curve whose strict transform, on the surface given by the point blowing-up sequence providing the exceptional divisor $E_{i}$ that defines the ideal, is regular and meets $E_{i}$ transversally at a nonsingular point. Also, notice that in the previous sentence "suitable chosen" means that the curves meet $E_{i}$ at different points.

\section{Proofs}

This section is devoted to prove the results that we have stated in the previous one. To this purpose, in each subsection, we will introduce some notation and prove some properties which will be necessary to deduce our results. Notation and lemmas in Subsection $3 \cdot 1$ are also useful for Subsection $3 \cdot 2$.

\subsection{Proposition 2.3: auxiliary results and proof}

We start this section with a lemma which is deduced from [15, Section 3] and will be a key tool for our proofs.

LEMMA 3.1. Let $h_{1}$ and $h_{2}$ be two irreducible elements of $R$ such that $\pi$ is a common log resolution of the curves $H_{1}$ and $H_{2}\left(H_{1} \neq H_{2}\right)$ that they define. Set $I\left(h_{1}, h_{2}\right)$ (respectively, $\left.\left(h_{1} \mid h_{2}\right)=(q, c)\right)$ the intersection multiplicity (respectively, contact pair) of $h_{1}$ and $h_{2}$. Then:

(a) $q \geqslant 1$ if, and only if, $\bar{\beta}_{0}^{h_{1}} \bar{\beta}_{1}^{h_{2}}=\bar{\beta}_{1}^{h_{1}} \bar{\beta}_{0}^{h_{2}} \leqslant I\left(h_{1}, h_{2}\right)$.

(b) If $q=1$ and $c=0$, then $I\left(h_{1}, h_{2}\right)=\bar{\beta}_{0}^{h_{1}} \bar{\beta}_{1}^{h_{2}}=\bar{\beta}_{1}^{h_{1}} \bar{\beta}_{0}^{h_{2}}$.

(c) If $q=0$ and $c \leqslant \min \left\{l_{0}^{h_{1}}, l_{0}^{h_{2}}\right\}$, then $I\left(h_{1}, h_{2}\right)=c \bar{\beta}_{0}^{h_{1}} \bar{\beta}_{0}^{h_{2}}$.

(d) If $q=0$ and $c=\min \left\{l_{0}^{h_{1}}+1, l_{0}^{h_{2}}+1\right\}$, then $I\left(h_{1}, h_{2}\right)=\min \left\{\bar{\beta}_{0}^{h_{1}} \bar{\beta}_{1}^{h_{2}}, \bar{\beta}_{1}^{h_{1}} \bar{\beta}_{0}^{h_{2}}\right\}$.

With the previous notation, for every curvette $\varphi$ at a point of $\mathcal{F}$, we consider the following subsets of $\mathbb{J}_{r}:=\{1,2, \ldots, r\}$ : 
- $J_{1}(\varphi):=\left\{s \in \mathbb{J}_{r} \mid\left(\varphi \mid f_{s}\right)=(0, c)\right.$ with $c=\min \left\{l_{0}^{\varphi}+1, l_{0}^{s}+1\right\}$ and $\left.\frac{\bar{\beta}_{1}^{s}}{\bar{\beta}_{0}^{s}}<\frac{\bar{\beta}_{1}^{\varphi}}{\bar{\beta}_{0}^{\varphi}}\right\}$,

- $J_{2}(\varphi):=\left\{s \in \mathbb{J}_{r} \mid\left(\varphi \mid f_{s}\right)=(0, c)\right.$ with $c=\min \left\{l_{0}^{\varphi}+1, l_{0}^{s}+1\right\}$ and $\left.\frac{\bar{\beta}_{1}^{s}}{\bar{\beta}_{0}^{s}}>\frac{\bar{\beta}_{1}^{\varphi}}{\bar{\beta}_{0}^{\varphi}}\right\}$,

- $J_{3}(\varphi):=\left\{s \in \mathbb{J}_{r} \mid\left(\varphi \mid f_{s}\right)=(1, c)\right.$ for some $\left.c \geqslant 0\right\}$,

- $J_{4}(\varphi):=\left\{s \in \mathbb{J}_{r} \mid\left(\varphi \mid f_{s}\right)=(0, c)\right.$ with $\left.c \leqslant \min \left\{l_{0}^{\varphi}, l_{0}^{s}\right\}\right\}$.

Notice that the non-empty elements of $\left\{J_{1}(\varphi), J_{2}(\varphi), J_{3}(\varphi), J_{4}(\varphi)\right\}$ define a partition of $\mathbb{J}_{r}$. If $\varphi$ defines a nonsingular curve then $J_{4}(\varphi)=J_{4,1}(\varphi) \cup J_{4,2}(\varphi)$, where

- $J_{4,1}(\varphi):=\left\{s \in \mathbb{J}_{r} \mid\left(\varphi \mid f_{s}\right)=(0, c)\right.$ with $c<l_{0}^{\varphi}$ and $\left.c \leqslant l_{0}^{s}\right\}$ and

- $J_{4,2}(\varphi):=\left\{s \in \mathbb{J}_{r} \mid\left(\varphi \mid f_{s}\right)=(0, c)\right.$ with $\left.c=l_{0}^{\varphi} \leqslant l_{0}^{s}\right\}$.

The following lemmas provide some properties that use and study the sets $J_{l}(\varphi)$. They will be needed for the development of our paper.

Lemma 3.2. Let $\varphi$ be a curvette at a free point $P_{j} \in \mathcal{F}$. Then $\varphi$ defines a nonsingular curve and $J_{2}(\varphi)=J_{3}(\varphi)=\emptyset$.

Proof. Since $P_{j}$ is a free point that belongs to $\mathcal{F}$ and $\varphi$ is transversal to $E_{j}, \varphi$ defines a nonsingular curve. Then, the equality $J_{3}(\varphi)=\emptyset$ is clear because there is no terminal satellite point for $\varphi$.

Reasoning by contradiction, assume that there exists $s \in\{1, \ldots, r\}$ such that $s \in$ $J_{2}(\varphi)$. Then $C_{s}$ is a singular curve and, taking into account that $\varphi$ is nonsingular, we have that $\left(\varphi \mid f_{s}\right)=(0, c)$ with $c=l_{0}^{s}+1$ and $\bar{\beta}_{1}^{s} / \bar{\beta}_{0}^{s}>c$. That is, $c$ is the number of free points in $\mathcal{C}_{s} \cap \mathcal{F}$ and $\bar{\beta}_{1}^{s}>c \bar{\beta}_{0}^{s}$. But this contradicts Noether's formula because $\bar{\beta}_{0}^{s}$ is the multiplicity of $C_{s}, \bar{\beta}_{1}^{s}=I\left(\varphi, f_{s}\right)$ and $C_{s}$ is singular.

Lemma 3.3. With the above notations, let $P_{j} \in \mathcal{F}$ and let $\varphi$ be a curvette at $P_{j}$.

(a) If $P_{j}=P_{t_{i}} \in \mathcal{T}$, then

$$
\mathbf{v}_{t_{i}}^{<}=\left\{\mathbf{a}_{s} \mid s \in J_{1}(\varphi) \cup J_{4}(\varphi)\right\} \quad \text { and } \quad \mathbf{v}_{t_{i}}^{\geqslant}=\left\{\mathbf{a}_{s} \mid s \in J_{2}(\varphi) \cup J_{3}(\varphi)\right\} .
$$

(b) If $P_{j}$ is free, then

$$
\mathbf{v}_{j}^{<}=\left\{\mathbf{a}_{s} \mid s \in J_{1}(\varphi) \cup J_{4,1}(\varphi)\right\} \quad \text { and } \quad \mathbf{v}_{j}^{\geqslant}=\left\{\mathbf{a}_{s} \mid s \in J_{4,2}(\varphi)\right\} .
$$

Proof. For any couple of irreducible elements $h_{1}, h_{2} \in R\left(H_{1} \neq H_{2}\right)$, we define

$$
H\left(h_{1}, h_{2}\right):=\frac{I\left(h_{1}, h_{2}\right)}{\bar{\beta}_{0}^{h_{2}}} .
$$

In order to prove (a), first consider an index $s \in J_{1}(\varphi)$ and let $\varphi^{\prime}$ be a curvette at $P_{j}$ different from $\varphi$. Lemma $3 \cdot 1$ implies that

$$
H\left(\varphi, f_{s}\right)=\frac{\bar{\beta}_{1}^{s} \bar{\beta}_{0}^{\varphi}}{\bar{\beta}_{0}^{s}} \quad \text { and } \quad H\left(\varphi, \varphi^{\prime}\right)=\bar{\beta}_{1}^{\varphi} .
$$

Since $\bar{\beta}_{1}^{s} / \bar{\beta}_{0}^{s}<\bar{\beta}_{1}^{\varphi} / \bar{\beta}_{0}^{\varphi}$, the following inequality holds:

$$
H\left(\varphi, f_{s}\right)<H\left(\varphi, \varphi^{\prime}\right) .
$$

By a result stated in $[\mathbf{1 3}$, page 425], and proved in [16], the last inequality is equivalent to fact that if $\mathbf{v}_{k}$ is the vertex satisfying $\left[\mathbf{v}_{1}, \mathbf{v}_{j}\right] \cap\left[\mathbf{v}_{1}, \mathbf{a}_{s}\right]=\left[\mathbf{v}_{1}, \mathbf{v}_{k}\right]$ then $\mathbf{v}_{k}<\mathbf{v}_{j}$. Moreover, since $\left(\varphi \mid f_{s}\right)=(0, c)$ with $c=\min \left\{l_{0}^{\varphi}+1, l_{0}^{s}+1\right\}$, the process of construction of the dual graph $\Gamma(C)$ allows us to deduce that either $\mathbf{v}_{k}=\mathbf{v}_{j}$ or $\mathbf{v}_{k}=\mathbf{a}_{s}$. So, the 
unique possibility is $\mathbf{v}_{k}=\mathbf{a}_{s}$ and, therefore, $\mathbf{a}_{s} \in \mathbf{v}_{j}^{<}$. We notice that, although the mentioned result of $[\mathbf{1 3}, \mathbf{1 6}]$ is stated over the complex numbers, its proof depends only on the Hamburger-Noether expansions of the curves, which are independent of the characteristic of the ground field.

Assume now that $s \in J_{2}(\varphi)$ and consider a curvette $\psi$ at the point $P_{r}$ such that $\mathbf{a}_{s}$ is a label of $\mathbf{v}_{r}$. Then, the inequality

$$
H\left(f_{s}, \varphi\right)<H\left(f_{s}, \psi\right)
$$

holds because $H\left(f_{s}, \varphi\right)=\bar{\beta}_{1}^{\varphi} \bar{\beta}_{0}^{s} / \bar{\beta}_{0}^{\varphi}, H\left(f_{s}, \psi\right)=\bar{\beta}_{1}^{s}$ and $\bar{\beta}_{1}^{\varphi} / \bar{\beta}_{0}^{\varphi}<\bar{\beta}_{1}^{s} / \bar{\beta}_{0}^{s}$. Also, again by $\left[\mathbf{1 3}\right.$, page 425], $\mathbf{v}_{k}<\mathbf{a}_{s}$, where $\mathbf{v}_{k}$ is the above mentioned vertex. Since $\left(\varphi \mid f_{s}\right)=(0, c)$ with $c=\min \left\{l_{0}^{\varphi}+1, l_{0}^{s}+1\right\}, \mathbf{v}_{k}$ is either $\mathbf{v}_{j}$ or $\mathbf{a}_{s}$. So we have that $\mathbf{v}_{k}=\mathbf{v}_{j}$ and, as a consequence, $\mathbf{a}_{s} \in \mathbf{v}_{j}^{\geqslant}$.

When $s \in J_{3}(\varphi)$, it is clear that $\mathbf{v}_{j} \leqslant \mathbf{a}_{s}$ because $\varphi$ and $f_{s}$ share its minimum terminal satellite point (that is $P_{j}=P_{t_{i}}$ ).

Finally, assume that $s \in J_{4}(\varphi)$ and suppose that $\mathbf{a}_{s} \notin \mathbf{v}_{j}^{<}$. This means that $\mathbf{v}_{j} \leqslant \mathbf{a}_{s}$ and, in fact, $\mathbf{v}_{j}<\mathbf{a}_{s}$ (because $s \notin J_{3}(\varphi)$ ). By [13, page 425] we have that $H\left(\varphi, \varphi^{\prime}\right)<$ $H\left(\varphi, f_{s}\right), \varphi^{\prime}$ being also a curvette at $P_{j}$ different from $\varphi$. This implies, using Lemma $3 \cdot 1$, that $c>\bar{\beta}_{1}^{\varphi} / \bar{\beta}_{0}^{\varphi}, c$ being the value such that $\left(\varphi \mid f_{s}\right)=(0, c)$. This is a contradiction and, thus, $\mathbf{a}_{s} \in \mathbf{v}_{j}^{<}$.

Therefore, the previous paragraphs and the fact that $\left\{J_{1}(\varphi), J_{2}(\varphi), J_{3}(\varphi), J_{4}(\varphi)\right\}$ is a partition of $\mathbb{J}_{r}$ conclude the proof of (a).

With respect to (b), our reasoning is analogous because the inclusion $J_{1}(\varphi) \subseteq \mathbf{v}_{j}^{<}$ (respectively, $J_{4,1}(\varphi) \subseteq \mathbf{v}_{j}^{<}, J_{4,2}(\varphi) \subseteq \mathbf{v}_{j}^{\geqslant}$) can be proved in a similar way to the proof of $J_{1}(\varphi) \subseteq \mathbf{v}_{j}^{<}$(respectively, $J_{4}(\varphi) \subseteq \mathbf{v}_{j}^{<}, J_{2}(\varphi) \cup J_{3}(\varphi) \subseteq \mathbf{v}_{j}^{\geqslant}$) of (a).

Given a curvette $\varphi$ at a point of $\mathcal{F}$, the sets $J_{3}(\varphi)$ and $J_{4}(\varphi)$ (or $J_{4,1}(\varphi)$ and $J_{4,2}(\varphi)$ if $\varphi$ is nonsingular) are easy to compute only from the proximity graph of $C$. The following result, that is a straightforward consequence of the two previous lemmas, will allow us to detect, only by inspection of the proximity and dual graphs of $C$, the elements of the sets $J_{k}(\varphi), k \in\{1,2\}$.

LEMma 3.4. Let $\varphi$ be a curvette at a point $P_{j} \in \mathcal{F}$ and let $s \in\{1, \ldots, r\}$.

(a) $s \in J_{1}(\varphi)$ if and only if $\left(\varphi \mid f_{s}\right)=(0, c)$ with $c=\min \left\{l_{0}^{\varphi}+1, l_{0}^{s}+1\right\}$ and $\mathbf{a}_{s} \in \mathbf{v}_{j}^{<}$.

(b) $s \in J_{2}(\varphi)$ if and only if $\left(\varphi \mid f_{s}\right)=(0, c)$ with $c=\min \left\{l_{0}^{\varphi}+1, l_{0}^{s}+1\right\}$ and $\mathbf{a}_{s} \in \mathbf{v}_{j}^{\geqslant}$.

Example 3.5 . Denote by $\varphi_{j}$ a curvette at the point $P_{j}$ in the constellation $\mathcal{C}$ of Example 1.1.A, $1 \leqslant j \leqslant 17$. The partitions of $\mathbb{J}_{8}$ defined the curvettes $\varphi_{j}$ at the points in $\mathcal{T} \cup \mathcal{S}$ are given by the following sets:

$$
\begin{aligned}
& \varphi_{2} \quad J_{1}\left(\varphi_{2}\right)=\emptyset, J_{2}\left(\varphi_{2}\right)=\emptyset, J_{3}\left(\varphi_{2}\right)=\emptyset, J_{4,1}\left(\varphi_{2}\right)=\emptyset, J_{4,2}\left(\varphi_{2}\right)=\{1,2,3,4,5,6,7,8\} . \\
& \varphi_{4} J_{1}\left(\varphi_{4}\right)=\{1,2\}, J_{2}\left(\varphi_{4}\right)=\emptyset, J_{3}\left(\varphi_{4}\right)=\emptyset, J_{4,1}\left(\varphi_{4}\right)=\{5,8\}, J_{4,2}\left(\varphi_{4}\right)=\{3,4,6,7\} . \\
& \varphi_{7} \quad J_{1}\left(\varphi_{7}\right)=\emptyset, \quad J_{2}\left(\varphi_{7}\right)=\{2,3,4,6,7\}, \quad J_{3}\left(\varphi_{7}\right)=\{1\}, \quad J_{4}\left(\varphi_{7}\right)=\{5,8\} . \\
& \varphi_{8} \quad J_{1}\left(\varphi_{8}\right)=\{1\}, \quad J_{2}\left(\varphi_{8}\right)=\{3,4,6,7\}, \quad J_{3}\left(\varphi_{8}\right)=\{2\}, \quad J_{4}\left(\varphi_{8}\right)=\{5,8\} . \\
& \varphi_{11} \quad J_{1}\left(\varphi_{11}\right)=\{1,2\}, \quad J_{2}\left(\varphi_{11}\right)=\{4\}, \quad J_{3}\left(\varphi_{11}\right)=\{3\}, \quad J_{4}\left(\varphi_{11}\right)=\{5,8,6,7\} . \\
& \varphi_{13} \quad J_{1}\left(\varphi_{13}\right)=\{1,2,3\}, \quad J_{2}\left(\varphi_{13}\right)=\emptyset, \quad J_{3}\left(\varphi_{13}\right)=\{4\}, \quad J_{4}\left(\varphi_{13}\right)=\{5,8,6,7\} . \\
& \varphi_{15} J_{1}\left(\varphi_{15}\right)=\{1,2\}, \quad J_{2}\left(\varphi_{15}\right)=\emptyset, \quad J_{3}\left(\varphi_{15}\right)=\emptyset, \quad J_{4,1}\left(\varphi_{15}\right)=\{3,4,5,8\}, \\
& J_{4,2}\left(\varphi_{15}\right)=\{6,7\} \text {. } \\
& \varphi_{17} J_{1}\left(\varphi_{17}\right)=\emptyset, \quad J_{2}\left(\varphi_{17}\right)=\{8\}, \quad J_{3}\left(\varphi_{17}\right)=\{5\}, \quad J_{4}\left(\varphi_{17}\right)=\{1,2,3,4,6,7\} .
\end{aligned}
$$


Next two lemmas will allow us to prove Proposition 2.3 and to show expressions of certain candidates for log-canonical threshold.

Lemma 3.6. If $\varphi$ is a curvette at $P_{j} \in \mathcal{F}$, then $\bar{\alpha}_{j}=\frac{\bar{\beta}_{0}^{\varphi}+\bar{\beta}_{1}^{\varphi}}{\sum_{i=1}^{r} I\left(\varphi, f_{i}\right)}$.

Proof. Consider the proximity matrix $\mathbf{P}$ of the configuration $\mathcal{C}$ associated with the minimal $\log$ resolution of the curve $C$. As we have said, the vector $\left(a_{1}, a_{2}, \ldots, a_{m}\right)$ used in $(1 \cdot 2)$ can be computed as follows: $\left(a_{1}, \ldots, a_{m}\right)^{t}=\mathbf{P}^{-1}(1, \ldots, 1)^{t}$. The reason comes from the fact that $K_{X / X_{0}}=\sum_{j=1}^{m} E_{j}^{*}$. Moreover, the entries of the $j$ th row of $\mathbf{P}^{-1}$, $1 \leqslant j \leqslant m$, are the multiplicities at the points of $\mathcal{C}$ of (the strict transforms of) a curvette at $P_{j}$. Therefore, it holds that $a_{j}$ is the sum of the multiplicities at the points of $\mathcal{C}$ of the strict transforms of a curvette at $P_{j}$. If the curve that $\varphi$ provides, $C_{\varphi}$, is not regular, then the equality $a_{j}+1=\bar{\beta}_{0}^{\varphi}+\bar{\beta}_{1}^{\varphi}$ follows from [12, Lemma 3.3.6] and the fact that $\bar{\beta}_{0}^{\varphi}$ and $\bar{\beta}_{1}^{\varphi}$ coincide with the first two characteristic exponents of $C_{\varphi}[\mathbf{1 2}$, Proposition 4.3.5]. Otherwise $\bar{\beta}_{0}^{\varphi}=1$ and $\bar{\beta}_{1}^{\varphi}$ is, as we have defined above, the number of points through which the strict transforms of $C_{\varphi}$ pass. Then, it is clear that the equality $a_{j}+1=\bar{\beta}_{0}^{\varphi}+\bar{\beta}_{1}^{\varphi}$ is also true.

We have just proved that the numerator of $\bar{\alpha}_{\varphi}$ is as stated. With respect to the denominator, it holds that

$$
\left(b_{1}, b_{2}, \ldots, b_{m}\right)^{t}:=\mathbf{P}^{-1}\left(\mathbf{n}_{1}, \mathbf{n}_{2}, \ldots, \mathbf{n}_{m}\right)^{t}=\sum_{i=1}^{r} \mathbf{P}^{-1}\left(\mathbf{n}_{i 1}, \mathbf{n}_{i 2}, \ldots, \mathbf{n}_{i m}\right)^{t},
$$

where $\mathbf{n}_{i j}$ denotes the multiplicity at $P_{j}$ of the strict transform of $C_{i}$. By Noether's formula, the $j$ th component of the vector $\mathbf{P}^{-1}\left(\mathbf{n}_{i 1}, \ldots, \mathbf{n}_{i m}\right)^{t}$ is the intersection multiplicity between $f_{i}$ and a curvette $\varphi$ at $P_{j}$. Therefore $b_{j}=\sum_{i=1}^{r} I\left(\varphi, f_{i}\right)$, which concludes the proof.

LEMma 3·7. The candidates for log-canonical threshold given by the minimum terminal satellite points $P_{t_{i}}, 1 \leqslant i \leqslant n$, can be computed as

$$
\bar{\alpha}_{t_{i}}=\frac{\bar{\beta}_{0}^{i}+\bar{\beta}_{1}^{i}}{\sum_{s=1}^{r} \delta_{i s}},
$$

where the values $\delta_{i s}$ are defined as in the statement of Theorem 2.13.

Proof. Let $\varphi$ be a curvette at $P_{t_{i}}$ and set $e_{1}^{i}:=\operatorname{gcd}\left(\bar{\beta}_{0}^{i}, \bar{\beta}_{1}^{i}\right)$. Using Noether's formula and Lemma $3 \cdot 1$, it is easy to deduce that

$$
\bar{\beta}_{0}^{\varphi}=\frac{\bar{\beta}_{0}^{i}}{e_{1}^{i}} \quad \text { and } \quad I\left(\varphi, f_{s}\right)=\frac{\delta_{i s}}{e_{1}^{i}} .
$$

Then, the result follows from Lemma $3 \cdot 6$.

Now we are ready to give a proof of Proposition 2.3. Take a curvette $\varphi$ at a point $P_{k} \in \mathcal{C} \backslash \mathcal{F}$ and let $i_{0} \in\{1,2, \ldots, n\}$ such that $P_{k} \in \mathcal{C}_{i_{0}}$. It is enough to show that $\bar{\alpha}_{k} \geqslant \bar{\alpha}_{t_{i_{0}}}$. Let $\psi$ be a curvette at $P_{t_{i_{0}}}$. By the proof of Lemma $3 \cdot 6$, there exists a positive integer $\epsilon$ such that

$$
\bar{\alpha}_{k}=\frac{\bar{\beta}_{1}^{\varphi}+\bar{\beta}_{0}^{\varphi}+\epsilon}{\sum_{s=1}^{r} I\left(\varphi, f_{s}\right)}
$$

and

$$
\bar{\alpha}_{t_{i_{0}}}=\frac{\bar{\beta}_{1}^{\psi}+\bar{\beta}_{0}^{\psi}}{\sum_{s=1}^{r} I\left(\psi, f_{s}\right)} .
$$


Some straightforward computations show that the inequality $\bar{\alpha}_{k} \geqslant \bar{\alpha}_{t_{i_{0}}}$ holds if the inequality

$$
\left(\bar{\beta}_{1}^{\varphi}+\bar{\beta}_{0}^{\varphi}+\epsilon\right) I\left(\psi, f_{s}\right) \geqslant\left(\bar{\beta}_{1}^{\psi}+\bar{\beta}_{0}^{\psi}\right) I\left(\varphi, f_{s}\right)
$$

is true for all $s \in \mathbb{J}_{r}$.

Let us consider the partition of $\mathbb{J}_{r},\left\{J_{l}(\psi)\right\}_{l=1}^{4}$, given at the top of this section. Assume first $s \in J_{3}(\psi)$ and thus $P_{t_{i_{0}}}=P_{t_{s}}$. Since $\pi$ is a $\log$ resolution of the curve $C_{s}$, it happens $\pi^{*} C_{s}=\tilde{C}_{s}+\sum_{j=1}^{m} b_{j s} E_{j}$ for some nonnegative integers $b_{j s}$ and the quotient $\bar{\alpha}_{k}^{\prime}:=\frac{\bar{\beta}_{1}^{\varphi}+\bar{\beta}_{0}^{\varphi}+\epsilon}{I\left(\varphi, f_{s}\right)}$ coincides with the candidate $\frac{a_{k}+1}{b_{k}}$ for log-canonical threshold of $C_{s}$. But, by the proof of Lemma $3 \cdot 7$, the quotient $\bar{\alpha}_{t_{i_{0}}}^{\prime}:=\left(\bar{\beta}_{1}^{\psi}+\bar{\beta}_{0}^{\psi}\right) / I\left(\psi, f_{s}\right)$ coincides with

$$
\frac{\bar{\beta}_{0}^{s}+\bar{\beta}_{1}^{s}}{\bar{\beta}_{0}^{s} \bar{\beta}_{1}^{s}}=\frac{1}{\bar{\beta}_{0}^{s}}+\frac{1}{\bar{\beta}_{1}^{s}},
$$

which is the log-canonical threshold of $C_{s}$ (see $\left.[\mathbf{2 4}]\right)$. Therefore $\bar{\alpha}_{k}^{\prime} \geqslant \bar{\alpha}_{t_{i_{0}}}^{\prime}$ and, then, inequality $(3 \cdot 4)$ holds.

Now suppose that $s$ is in other set of the partition. By Lemma $3 \cdot 1$ it happens that

$$
I\left(\varphi, f_{s}\right):=\left\{\begin{array}{lll}
\bar{\beta}_{1}^{s} \bar{\beta}_{0}^{\varphi} & \text { if } & s \in J_{1}(\psi) \\
\bar{\beta}_{0}^{s} \bar{\beta}_{1}^{\varphi} & \text { if } & s \in J_{2}(\psi) \\
c \bar{\beta}_{0}^{s} \bar{\beta}_{0}^{\varphi} & \text { if } & s \in J_{4}(\psi),
\end{array}\right.
$$

where $c$ is such that $\left(\varphi \mid f_{s}\right)=(0, c)$. Writing $\psi$ instead of $\varphi$, we obtain an analogous formula which can be checked using the same argument.

Proposition $2 \cdot 3$ will be proved if we show that

$$
\left(\bar{\beta}_{1}^{\varphi}+\bar{\beta}_{0}^{\varphi}\right) I\left(\psi, f_{s}\right) \geqslant\left(\bar{\beta}_{1}^{\psi}+\bar{\beta}_{0}^{\psi}\right) I\left(\varphi, f_{s}\right)
$$

because this inequality implies that of $(3 \cdot 4)$. And $(3 \cdot 5)$ is equivalent to

$$
\frac{\bar{\beta}_{1}^{\varphi}}{\bar{\beta}_{0}^{\varphi}} \geqslant \frac{\bar{\beta}_{1}^{\psi}}{\bar{\beta}_{0}^{\psi}} \quad\left(\text { respectively, } \frac{\bar{\beta}_{1}^{\varphi}}{\bar{\beta}_{0}^{\varphi}} \leqslant \frac{\bar{\beta}_{1}^{\psi}}{\bar{\beta}_{0}^{\psi}}, \frac{\bar{\beta}_{1}^{\varphi}}{\bar{\beta}_{0}^{\varphi}} \geqslant \frac{\bar{\beta}_{1}^{\psi}}{\bar{\beta}_{0}^{\psi}}\right),
$$

whenever $s \in J_{1}(\psi)$ (respectively, $s \in J_{2}(\psi), s \in J_{4}(\psi)$ ), which concludes the proof since

$$
\frac{\bar{\beta}_{1}^{\varphi}}{\bar{\beta}_{0}^{\varphi}}=\frac{\bar{\beta}_{1}^{\psi}}{\bar{\beta}_{0}^{\psi}}
$$

It only remains to add that this equality is true because $\varphi$ and $\psi$ share its minimum terminal satellite point $P_{t_{i_{0}}}$.

\subsection{Theorem 2.13: auxiliary results and proof}

In this section we keep the notations introduced in the previous ones.

Firstly and by means of the following three lemmas, we will prove (1) of Theorem $2 \cdot 13$. The first lemma shows that $\sigma$ is an increasing function on the set $\mathcal{V}_{\mathcal{F}}$ (under the ordering $\leqslant$ defined before the definitions in $(2 \cdot 1))$ and it follows from the fact that $\mathbf{v}_{k_{1}}^{<} \subseteq \mathbf{v}_{k_{2}}^{<}$and $\mathbf{v}_{k_{2}}^{\geqslant} \subseteq \mathbf{v}_{k_{1}}^{\geqslant}$, whenever $\mathbf{v}_{k_{1}}, \mathbf{v}_{k_{2}} \in \mathcal{V}_{\mathcal{F}}$ and $\mathbf{v}_{k_{1}}<\mathbf{v}_{k_{2}}$.

LEMMA 3.8. Let $\mathbf{v}_{k_{1}}$ and $\mathbf{v}_{k_{2}}$ be two vertices in $\mathcal{V}_{\mathcal{F}}$ such that $\mathbf{v}_{k_{1}}<\mathbf{v}_{k_{2}}$. Then $\sigma\left(\mathbf{v}_{k_{1}}\right) \leqslant$ $\sigma\left(\mathbf{v}_{k_{2}}\right)$.

Lemma 3.9. Let $\mathbf{v}_{k} \in \mathcal{V}$ and let $D:=\left\{\mathbf{v}_{j} \in \mathcal{V}_{\mathcal{F}} \backslash\left\{\mathbf{v}_{k}\right\} \mid \mathbf{v}_{j} \geqslant \mathbf{v}_{k}\right.$ and $\left.\sigma\left(\mathbf{v}_{j}\right)<0\right\}$. Then, $D$ has, at most, one maximal element (with respect to the order relation $\leqslant$ ). 
Proof. Reasoning by contradiction, assume the existence of two maximal elements $\mathbf{v}_{j_{1}}, \mathbf{v}_{j_{2}}$ in $D$. One has that

$$
\sigma\left(\mathbf{v}_{j_{a}}\right)=\sum_{\mathbf{a}_{i} \in \mathbf{v}_{j_{a}}^{<}} c_{j_{a} i} \bar{\beta}_{0}^{i}-\sum_{\mathbf{a}_{i} \in \mathbf{v}_{j_{a}}^{>}} \bar{\beta}_{0}^{i}<0,
$$

for $a=1,2$.

On the one hand we have that $\mathbf{v}_{j_{1}}^{\geqslant} \subseteq \mathbf{v}_{j_{2}}^{<}$and, therefore,

$$
\sum_{\mathbf{a}_{i} \in \mathbf{v}_{j_{2}}^{\geqslant}} \bar{\beta}_{0}^{i}>\sum_{\mathbf{a}_{i} \in \mathbf{v}_{j_{2}}^{<}} c_{j_{2} i} \bar{\beta}_{0}^{i} \geqslant \sum_{\mathbf{a}_{i} \in \mathbf{v}_{j_{1}}^{\geqslant}} c_{j_{2} i} \bar{\beta}_{0}^{i} \geqslant \sum_{\mathbf{a}_{i} \in \mathbf{v}_{j_{1}}^{\geqslant}} \bar{\beta}_{0}^{i} .
$$

On the other hand, by a symmetric reasoning, it holds that

$$
\sum_{\mathbf{a}_{i} \in \mathbf{v}_{j_{1}}^{\geqslant}} \bar{\beta}_{0}^{i}>\sum_{\mathbf{a}_{i} \in \mathbf{v}_{j_{2}}^{\geqslant}} \bar{\beta}_{0}^{i}
$$

because $\mathbf{v}_{j_{2}}^{\geqslant} \subseteq \mathbf{v}_{j_{1}}^{<}$, which concludes the proof.

Lemma 3.10. There exists a vertex $\mathbf{v}_{k} \in \mathcal{V}_{\mathcal{F}}$ satisfying the conditions:

(a) $\sigma\left(\mathbf{v}_{j}\right)<0$ for all $\mathbf{v}_{j} \in\left[\mathbf{v}_{1}, \mathbf{v}_{k}\right]$ and

(b) $\sigma\left(\mathbf{v}_{j}\right) \geqslant 0$ for all $\mathbf{v}_{j} \in \mathcal{V}_{\mathcal{F}} \backslash\left[\mathbf{v}_{1}, \mathbf{v}_{k}\right]$.

Proof. The result follows from the two preceding lemmas and the fact that $\sigma\left(\mathbf{v}_{1}\right)<0$ because $\mathbf{v}_{1}^{<}=\emptyset$.

Now we are ready to prove Part (1) of Theorem 2.13. To do it, we need to define the concept of consecutive vertices in $\mathcal{V} \cup \mathcal{V}_{\text {end }}$.

Definition 3.11. We will say that two vertices $\mathbf{v}_{k_{1}}, \mathbf{v}_{k_{2}} \in \mathcal{V} \cup \mathcal{V}_{\text {end }}$ are consecutive if $\mathbf{v}_{k_{1}}<\mathbf{v}_{k_{2}}$ and $] \mathbf{v}_{k_{1}}, \mathbf{v}_{k_{2}}[\cap \mathcal{V}=\emptyset$.

Consider the vertex $\mathbf{v}_{k} \in \mathcal{V}_{\mathcal{F}}$ given by Lemma 3.10. If $\mathbf{v}_{1} \notin \mathcal{V}$ then the adapted degree of $\mathbf{v}_{1}$ is 2 and, therefore, there is no arrow labeling it; this implies that $\sigma\left(\mathbf{v}_{2}\right)=\sigma\left(\mathbf{v}_{1}\right)<0$ and, then, $\mathbf{v}_{k} \neq \mathbf{v}_{1}$.

Also, we claim that $\mathbf{v}_{k}$ cannot be a vertex in $\mathcal{V}_{\text {end }} \backslash \mathcal{V}$. Indeed, reasoning by contradiction, assume that $\mathbf{v}_{k} \in \mathcal{V}_{\text {end }} \backslash \mathcal{V}$. Then $\mathbf{v}_{k}$ is a vertex whose adapted degree is, at most, 2. This implies that $\mathbf{v}_{k}$ has, at most, one arrow $\mathbf{a}_{i}$ as a label (recall that $\mathbf{v}_{k} \neq \mathbf{v}_{1}$ ); moreover, $C_{i}$ must be a nonsingular curve since $P_{k}$ is a free point in $\mathcal{F}$ and, therefore, $\bar{\beta}_{0}^{i}=1$. Hence $\sigma\left(\mathbf{v}_{k}\right) \geqslant \sum_{\mathbf{a}_{s} \in \mathbf{v}_{k}^{<}} c_{k s} \bar{\beta}_{0}^{s}-1 \geqslant 0$ and this gives the desired contradiction.

So, if $\mathbf{v}_{k}$ belongs to $\mathcal{V}_{\text {end }}$ then it must belong to $\mathcal{V}$.

Finally, we are going to show that $\mathbf{v}_{k}$ belongs to $\mathcal{V}$, which proves (1) of Theorem $2 \cdot 13$ because $\mathbf{v}_{k}$ satisfies the conditions (a) and (b) given in the theorem's statement. Indeed, reasoning again by contradiction assume that $\mathbf{v}_{k} \notin \mathcal{V}$. Then there exist two consecutive vertices, $\mathbf{v}_{j_{1}}, \mathbf{v}_{j_{2}}$ in $\mathcal{V} \cup \mathcal{V}_{\text {end }}$, such that $\left.\mathbf{v}_{k} \in\right] \mathbf{v}_{j_{1}}, \mathbf{v}_{j_{2}}$ [ (notice that, by the above proved claim, $\left.\mathbf{v}_{k} \notin \mathcal{V}_{\text {end }}\right)$. Thus $\sigma\left(\mathbf{v}_{k}\right)=\sigma\left(\mathbf{v}_{j_{2}}\right)$ because $\sigma$ is constant along the path $\left.] \mathbf{v}_{j_{1}}, \mathbf{v}_{j_{2}}\right]$ (see Remark 2.12) which contradicts Condition (b) of Lemma 3.10.

Now, we are going to prove (2) of Theorem 2.13. The following lemma will be of importance for our proof.

LEMma 3.12. Let $\mathbf{v}_{k_{1}}, \mathbf{v}_{k_{2}}$ be two consecutive vertices of $\mathcal{V} \cup \mathcal{V}_{\mathrm{end}}$. It holds that: 
(a) If $\sigma\left(\mathbf{v}_{k_{2}}\right) \leqslant 0$, then $\bar{\alpha}_{j} \geqslant \bar{\alpha}_{k_{2}}$ for all $j$ such that $\mathbf{v}_{j} \in\left[\mathbf{v}_{k_{1}}, \mathbf{v}_{k_{2}}\right]$.

(b) If $\sigma\left(\mathbf{v}_{k_{2}}\right) \geqslant 0$, then $\bar{\alpha}_{j} \geqslant \bar{\alpha}_{k_{1}}$ for all $j$ such that $\mathbf{v}_{j} \in\left[\mathbf{v}_{k_{1}}, \mathbf{v}_{k_{2}}\right]$.

Proof. Let $\psi_{1}$ (respectively, $\psi_{2}$ ) be a curvette at $P_{k_{1}}$ (respectively, $P_{k_{2}}$ ).

If $l \in\{1,2\}$ and $\mathbf{v}_{k_{l}} \in \mathcal{V}_{\mathcal{S}} \cup \mathcal{V}_{\text {end }}$ then, by Lemma $3 \cdot 2$, the non-empty sets in the family

$$
\left\{J_{1}\left(\psi_{l}\right), J_{4,1}\left(\psi_{l}\right), J_{4,2}\left(\psi_{l}\right)\right\}
$$

form a partition of $\mathbb{J}_{r}$. Taking into account Lemmas $3 \cdot 1$ and $3 \cdot 6$, it holds that

$$
\bar{\alpha}_{k_{l}}=\frac{d_{k_{l}}+1}{\sum_{s \in J_{4,1}\left(\psi_{l}\right)} c_{k_{l} s} \bar{\beta}_{0}^{s}+\sum_{s \in J_{1}\left(\psi_{l}\right)} \bar{\beta}_{1}^{s}+d_{k_{l}} \sum_{s \in J_{4,2}\left(\psi_{l}\right)} \bar{\beta}_{0}^{s}}
$$

where, for each $P_{e} \in \mathcal{F}, d_{e}$ denotes the cardinality of the set $\left[\mathbf{v}_{1}, \mathbf{v}_{l}\right] \cap \mathcal{V}_{\text {free }}$.

If, on the contrary, $\mathbf{v}_{k_{l}}=\mathbf{v}_{t_{i}} \in \mathcal{V}_{\mathcal{T}}$, then applying Lemmas 3.1 and 3.6 again, we have that

$$
\bar{\alpha}_{k_{l}}=\bar{\alpha}_{t_{i}}=\frac{\bar{\beta}_{0}^{\psi_{l}}+\bar{\beta}_{1}^{\psi_{l}}}{\sum_{s \in J_{1}\left(\psi_{l}\right)} \bar{\beta}_{0}^{\psi_{l}} \bar{\beta}_{1}^{s}+\sum_{s \in J_{2}\left(\psi_{l}\right) \cup J_{3}\left(\psi_{l}\right)} \bar{\beta}_{0}^{s} \bar{\beta}_{1}^{\psi_{l}}+\sum_{s \in J_{4}\left(\psi_{l}\right)} c_{k_{l} s} \bar{\beta}_{0}^{\psi_{l}} \bar{\beta}_{0}^{s}} .
$$

We will distinguish four cases:

Case 1: $\mathbf{v}_{k_{1}}, \mathbf{v}_{k_{2}} \in \mathcal{V}_{\mathcal{S}} \cup \mathcal{V}_{\text {end }}$. Let us consider a vertex $\mathbf{v}_{j} \in\left[\mathbf{v}_{k_{1}}, \mathbf{v}_{k_{2}}\right]$ and let $\varphi$ be a curvette at $P_{j}$. Clearly, $P_{j}$ is free and $\varphi$ is smooth. Moreover $I\left(\varphi, f_{s}\right) \leqslant I\left(\psi_{2}, f_{s}\right)$ for all $s \in \mathbb{J}_{r}$ and, by Lemma $3 \cdot 1, I\left(\varphi, f_{s}\right)=d_{j} \bar{\beta}_{0}^{s}$ whenever $s \in J_{4,2}\left(\psi_{2}\right)$. Therefore

$$
\bar{\alpha}_{j} \geqslant \bar{\alpha}_{j}^{\prime}:=\frac{d_{j}+1}{\sum_{s \in J_{4,1}\left(\psi_{2}\right)} c_{k_{2} s} \bar{\beta}_{0}^{s}+\sum_{s \in J_{1}\left(\psi_{2}\right)} \bar{\beta}_{1}^{s}+d_{j} \sum_{s \in J_{4,2}\left(\psi_{2}\right)} \bar{\beta}_{0}^{s}} .
$$

Using (3.6), straightforward computations show that the inequality $\bar{\alpha}_{j}^{\prime} \geqslant \bar{\alpha}_{k_{2}}$ holds if and only if

$$
\left(d_{j}-d_{k_{2}}\right)\left(\sum_{s \in J_{4,1}\left(\psi_{2}\right)} c_{k_{2} s} \bar{\beta}_{0}^{s}+\sum_{s \in J_{1}\left(\psi_{2}\right)} \bar{\beta}_{1}^{s}-\sum_{s \in J_{4,2}\left(\psi_{2}\right)} \bar{\beta}_{0}^{s}\right) \geqslant 0
$$

and, by Lemma $3 \cdot 3$, this inequality can be written in the following form: $\left(d_{j}-d_{k_{2}}\right) \sigma\left(\mathbf{v}_{k_{2}}\right) \geqslant$ 0 . Since $d_{j} \leqslant d_{k_{2}}$, we have that $\bar{\alpha}_{j} \geqslant \bar{\alpha}_{k_{2}}$ if $\sigma\left(\mathbf{v}_{k_{2}}\right) \leqslant 0$. So, we have proved Part (a) in Case 1.

To prove (b), let us consider a vertex $\mathbf{v}_{j}$ and a curvette $\varphi$ as above. The facts $J_{4,2}\left(\psi_{2}\right) \subseteq$ $J_{4,2}\left(\psi_{1}\right)$ and $c_{k_{1} s}=d_{k_{1}}$ for every $s \in \Delta:=J_{4,2}\left(\psi_{1}\right) \backslash J_{4,2}\left(\psi_{2}\right)$ allow us to write the equality $(3 \cdot 6)$ as

$$
\bar{\alpha}_{k_{1}}=\frac{d_{k_{1}}+1}{\sum_{s \in J_{4,1}\left(\psi_{1}\right) \cup \Delta} c_{k_{1} s} \bar{\beta}_{0}^{s}+\sum_{s \in J_{1}\left(\psi_{1}\right)} \bar{\beta}_{1}^{s}+d_{k_{1}} \sum_{s \in J_{4,2}\left(\psi_{2}\right)} \bar{\beta}_{0}^{s}} .
$$

On the other hand, from Lemma $3 \cdot 1$ one can deduce the following statements:

- If $s \in J_{4,1}\left(\psi_{1}\right)$ then $I\left(\varphi, f_{s}\right)=c_{k_{1} s} \bar{\beta}_{0}^{s}$, because $J_{4,1}\left(\psi_{1}\right) \subseteq J_{4,1}(\varphi)$ and $c_{j s}=c_{k_{1} s}$.

- If $s \in J_{1}\left(\psi_{1}\right)$ then $I\left(\varphi, f_{s}\right)=\bar{\beta}_{1}^{s}$, because $J_{1}\left(\psi_{1}\right) \subseteq J_{1}(\varphi)$.

- If $s \in J_{4,2}\left(\psi_{2}\right)$ then $I\left(\varphi, f_{s}\right)=d_{j} \bar{\beta}_{0}^{s}$, because $J_{4,2}\left(\psi_{2}\right) \subseteq J_{4,2}(\varphi)$.

- If $s \in \Delta$ then $I\left(\varphi, f_{s}\right)=d_{k_{1}} \bar{\beta}_{0}^{s}=c_{k_{1} s} \bar{\beta}_{0}^{s}$, because $\Delta \subseteq J_{4,1}(\varphi)$ and $c_{j s}=c_{k_{1} s}=d_{k_{1}}$.

Then, as a consequence of the previous statements and the fact that the non-empty sets in the family $\left\{J_{1}\left(\psi_{1}\right), J_{4,1}\left(\psi_{1}\right), J_{4,2}\left(\psi_{2}\right), \Delta\right\}$ are a partition of $\mathbb{J}_{r}$, it happens 


$$
\bar{\alpha}_{j}=\frac{d_{j}+1}{\sum_{s \in J_{4,1}\left(\psi_{1}\right) \cup \Delta} c_{k_{1} s} \bar{\beta}_{0}^{s}+\sum_{s \in J_{1}\left(\psi_{1}\right)} \bar{\beta}_{1}^{s}+d_{j} \sum_{s \in J_{4,2}\left(\psi_{2}\right)} \bar{\beta}_{0}^{s}} .
$$

From this equality and that in (3.8), one can deduce that $\bar{\alpha}_{j} \geqslant \bar{\alpha}_{k_{1}}$ if and only if

$$
\left(d_{j}-d_{k_{1}}\right)\left(\sum_{s \in J_{4,1}\left(\psi_{1}\right) \cup \Delta} c_{k_{1} s} \bar{\beta}_{0}^{s}+\sum_{s \in J_{1}\left(\psi_{1}\right)} \bar{\beta}_{1}^{s}-\sum_{s \in J_{4,2}\left(\psi_{2}\right)} \bar{\beta}_{0}^{s}\right) \geqslant 0 .
$$

Now, it is clear that $\mathbf{v}_{k_{2}}^{<}=\mathbf{v}_{k_{1}}^{<} \cup\left(\mathbf{v}_{k_{1}}^{\geqslant} \backslash \mathbf{v}_{k_{2}}^{\geqslant}\right)$and, using Lemma 3.3, one gets that

$$
\mathbf{v}_{k_{2}}^{<}=\left\{\mathbf{a}_{s} \mid s \in J_{4,1}\left(\psi_{1}\right) \cup \Delta \cup J_{1}\left(\psi_{1}\right)\right\} .
$$

Moreover $c_{k_{2} s}=c_{k_{1} s}$ for all $s \in J_{4,1}\left(\psi_{1}\right) \cup \Delta$. Thus the above inequality can be written as $\left(d_{j}-d_{k_{1}}\right) \sigma\left(\mathbf{v}_{k_{2}}\right) \geqslant 0$ and hence Part (b) in Case 1 holds because $d_{j} \geqslant d_{k_{1}}$.

Case 2: $\mathbf{v}_{k_{1}}, \mathbf{v}_{k_{2}} \in \mathcal{V}_{\mathcal{T}}$. Let us consider a vertex $\mathbf{v}_{j} \in\left[\mathbf{v}_{k_{1}}, \mathbf{v}_{k_{2}}\right]$ and a curvette $\varphi$ at $P_{j}$. By Lemma $3 \cdot 1$, it follows that $I\left(\varphi, f_{s}\right) \leqslant \min \left\{\bar{\beta}_{1}^{\varphi} \bar{\beta}_{0}^{s}, \bar{\beta}_{1}^{s} \bar{\beta}_{0}^{\varphi}\right\}$ for every $s \in \mathbb{J}_{r}$ and, moreover, $I\left(\varphi, f_{s}\right)=c_{k_{2} s} \bar{\beta}_{0}^{\varphi} \bar{\beta}_{0}^{s}$ for every $s \in J_{4}\left(\psi_{2}\right)$ (because the strict transforms of the curves $C_{s}$ and that defined by $\varphi$ pass through the same free points). Therefore

$$
\bar{\alpha}_{j} \geqslant \bar{\alpha}_{j}:=\frac{\bar{\beta}_{0}^{\varphi}+\bar{\beta}_{1}^{\varphi}}{\sum_{s \in J_{1}\left(\psi_{2}\right)} \bar{\beta}_{0}^{\varphi} \bar{\beta}_{1}^{s}+\sum_{s \in J_{2}\left(\psi_{2}\right) \cup J_{3}\left(\psi_{2}\right)} \bar{\beta}_{0}^{s} \bar{\beta}_{1}^{\varphi}+\sum_{s \in J_{4}\left(\psi_{2}\right)} c_{k_{2} s} \bar{\beta}_{0}^{\varphi} \bar{\beta}_{0}^{s}} .
$$

Taking into account $(3 \cdot 7)$, one can deduce that the inequality $\bar{\alpha}{ }_{j} \geqslant \bar{\alpha}_{k_{2}}$ holds if and only if

$$
\left(\bar{\beta}_{1}^{\varphi} \bar{\beta}_{0}^{\psi_{2}}-\bar{\beta}_{0}^{\varphi} \bar{\beta}_{1}^{\psi_{2}}\right)\left(\sum_{s \in J_{1}\left(\psi_{2}\right)} \bar{\beta}_{1}^{s}-\sum_{s \in J_{2}\left(\psi_{2}\right) \cup J_{3}\left(\psi_{2}\right)} \bar{\beta}_{0}^{s}+\sum_{s \in J_{4}\left(\psi_{2}\right)} c_{k_{2} s} \bar{\beta}_{0}^{s}\right) \geqslant 0 .
$$

Finally, $\mathbf{v}_{j} \leqslant \mathbf{v}_{k_{2}}$ implies $\bar{\beta}_{1}^{\varphi} / \bar{\beta}_{0}^{\varphi} \leqslant \bar{\beta}_{1}^{\psi_{2}} / \bar{\beta}_{0}^{\psi_{2}}$ (see the proof of Lemma 3.3) and since, by Lemma $3 \cdot 3$, the second factor of the left-hand side of the above inequality is $\sigma\left(\mathbf{v}_{k_{2}}\right)$, we have proved (a) in Case 2 because $\alpha_{j} \geqslant \alpha_{k_{2}}$ whenever $\sigma\left(\mathbf{v}_{k_{2}}\right) \leqslant 0$.

To prove Part (b) in this case, one only need to make an analogous reasoning. In fact, one has to use the expression of $\bar{\alpha}_{k_{1}}$ in (3.7) instead of $\bar{\alpha}_{k_{2}}$ and the facts that $J_{4}\left(\psi_{1}\right)=J_{4}\left(\psi_{2}\right)$ and $c_{k_{1} s}=c_{k_{2} s}$ for all $s \in J_{4}\left(\psi_{1}\right)$.

Case 3: $\mathbf{v}_{k_{1}} \in \mathcal{V}_{\mathcal{S}} \cup \mathcal{V}_{\text {end }}$ and $\mathbf{v}_{k_{2}} \in \mathcal{V}_{\mathcal{T}}$. Part (a) can be proved as in the previous case. To prove (b), keep the same notations and observe that $J_{1}\left(\psi_{2}\right)=\emptyset$. Now, $\psi_{1}$ is nonsingular (by Lemma 3.2) and

$$
\bar{\alpha}_{\psi_{1}}=\frac{d_{k_{1}}+1}{d_{k_{1}} \sum_{s \in J_{2}\left(\psi_{2}\right) \cup J_{3}\left(\psi_{2}\right)} \bar{\beta}_{0}^{s}+\sum_{s \in J_{4}\left(\psi_{2}\right)} c_{k_{1} s} \bar{\beta}_{0}^{s}},
$$

by Lemma 3·1. Moreover

$$
\bar{\alpha}_{j} \geqslant \bar{\alpha}_{j}^{(3)}:=\frac{\bar{\beta}_{0}^{\varphi}+\bar{\beta}_{1}^{\varphi}}{\sum_{s \in J_{2}\left(\psi_{2}\right) \cup J_{3}\left(\psi_{2}\right)} \bar{\beta}_{0}^{s} \bar{\beta}_{1}^{\varphi}+\sum_{s \in J_{4}\left(\psi_{2}\right)} c_{k_{1} s} \bar{\beta}_{0}^{\varphi} \bar{\beta}_{0}^{s}} .
$$


As a consequence $\bar{\alpha}_{j}^{(3)} \geqslant \bar{\alpha}_{\psi_{1}}$ if, and only if,

$$
\left(\bar{\beta}_{1}^{\varphi}-d_{k_{1}} \bar{\beta}_{0}^{\varphi}\right)\left(-\sum_{s \in J_{2}\left(\psi_{2}\right) \cup J_{3}\left(\psi_{2}\right)} \bar{\beta}_{0}^{s}+\sum_{s \in J_{4}\left(\psi_{2}\right)} c_{k_{1} s} \bar{\beta}_{0}^{s}\right) \geqslant 0 .
$$

And this inequality concludes the proof of (b) in this case after bearing in mind the following facts: $c_{k_{1} s}=c_{k_{2} s}$ for all $s \in J_{4}\left(\psi_{2}\right)$, the second factor of the left-hand side of the inequality is $\sigma\left(\mathbf{v}_{k_{2}}\right)$ by Lemma $3 \cdot 3$ and $d_{k_{1}} \leqslant \bar{\beta}_{1}^{\varphi} / \bar{\beta}_{0}^{\varphi}$ because the strict transforms of $\varphi$ pass through, at least, $d_{k_{1}}$ free points of $\mathcal{C}$.

Case 4: $\mathbf{v}_{k_{1}} \in \mathcal{V}_{\mathcal{T}}$ and $\mathbf{v}_{k_{2}} \in \mathcal{V}_{\mathcal{S}} \cup \mathcal{V}_{\text {end }}$. Reasoning in a similar way as we made for Part (b) of Case 1, the equality

$$
\bar{\alpha}_{j}=\frac{\bar{\beta}_{0}^{\varphi}+\bar{\beta}_{1}^{\varphi}}{\sum_{s \in J_{4,1}\left(\psi_{2}\right)} c_{k_{2} s} \bar{\beta}_{0}^{\varphi} \bar{\beta}_{0}^{s}+\sum_{s \in J_{1}\left(\psi_{2}\right)} \bar{\beta}_{0}^{\varphi} \bar{\beta}_{1}^{s}+\sum_{s \in J_{4,2}\left(\psi_{2}\right)} \bar{\beta}_{1}^{\varphi} \bar{\beta}_{0}^{s}}
$$

is a consequence of the following three statements:

- If $s \in J_{4,1}\left(\psi_{2}\right)$ then $I\left(\varphi, f_{s}\right)=c_{k_{2} s} \bar{\beta}_{0}^{\varphi} \bar{\beta}_{0}^{s}$, because $J_{4,1}\left(\psi_{2}\right) \subseteq J_{4}(\varphi)$ and $c_{j s}=c_{k_{2} s}$.

- If $s \in J_{1}\left(\psi_{2}\right)$ then $I\left(\varphi, f_{s}\right)=\bar{\beta}_{0}^{\varphi} \bar{\beta}_{1}^{s}$, because $J_{1}\left(\psi_{2}\right) \subseteq J_{1}(\varphi) \cup J_{3}(\varphi)$.

- If $s \in J_{4,2}\left(\psi_{2}\right)$ then $I\left(\varphi, f_{s}\right)=\bar{\beta}_{1}^{\varphi} \bar{\beta}_{0}^{s}$.

Thus using Equality (3.6) and Lemma 3.3, we show that $\bar{\alpha}_{j} \geqslant \bar{\alpha}_{k_{2}}$ if and only if $\left(\bar{\beta}_{1}^{\varphi}-d_{k_{2}} \bar{\beta}_{0}^{\varphi}\right) \sigma\left(\mathbf{v}_{k_{2}}\right) \geqslant 0$, which proves (a) in this case because $d_{k_{2}} \geqslant \bar{\beta}_{1}^{\varphi} / \bar{\beta}_{0}^{\varphi}$.

Finally, the proof of (b) is analogous to that of Case 2 and our lemma is proved.

EXAMPLE 3.13. As a complement of the above proof, we check some of the results there used for the curve given in Example 1-1.A. For a start, $\mathcal{V}=\mathcal{V}_{\mathcal{S}} \cup \mathcal{V}_{\mathcal{T}}$, where $\mathcal{V}_{\mathcal{S}}=\left\{\mathbf{v}_{2}, \mathbf{v}_{4}, \mathbf{v}_{15}\right\}$ and $\mathcal{V}_{\mathcal{T}}=\left\{\mathbf{v}_{7}, \mathbf{v}_{8}, \mathbf{v}_{11}, \mathbf{v}_{13}, \mathbf{v}_{17}\right\}$

Now consider the vertices $\mathbf{v}_{k_{1}}=\mathbf{v}_{4}$ and $\mathbf{v}_{k_{2}}=\mathbf{v}_{15}$, which are consecutive and both in $\mathcal{V}_{\mathcal{S}}$. Here $\psi_{1}=\varphi_{4}$ and $\psi_{2}=\varphi_{15} . J_{1}\left(\psi_{1}\right)=\{1,2\}, J_{4,1}\left(\psi_{1}\right)=\{5,8\}, J_{4,2}\left(\psi_{1}\right)=\{3,4,6,7\}$ and $J_{1}\left(\psi_{2}\right)=\{1,2\}, J_{4,1}\left(\psi_{2}\right)=\{3,4,5,8\}$ and $J_{4,2}\left(\psi_{2}\right)=\{6,7\}$. As we have said, both sets are a partition of $\mathbb{J}_{8}$. These vertices correspond to Part (b) in Case 1. Apart from $\psi_{1}$ and $\psi_{2}$, our lemma would also consider $\varphi=\varphi_{14}$ whose sets $J_{i}$ are $J_{1}(\varphi)=\{1,2\}$, $J_{4,1}(\varphi)=\{3,4,5,8\}$ and $J_{4,2}(\varphi)=\{6,7\}$. Moreover, $\Delta=J_{4,2}\left(\psi_{1}\right) \backslash J_{4,2}\left(\psi_{2}\right)=\{3,4\}$ and, as we have said, $\left\{J_{4,1}\left(\psi_{1}\right), J_{1}\left(\psi_{1}\right), J_{4,2}\left(\psi_{2}\right), \Delta\right\}$ is a partition of $\mathbb{J}_{8}$. Moreover, $J_{4,1}\left(\psi_{1}\right) \subseteq$ $J_{4,1}(\varphi), J_{1}\left(\psi_{1}\right) \subseteq J_{1}(\varphi), J_{4,2}\left(\psi_{2}\right) \subseteq J_{4,2}(\varphi)$ and $\Delta \subseteq J_{4,1}(\varphi)$, as we stated.

To finish, we consider a situation corresponding to Case 4 . It is $\mathbf{v}_{k_{1}}=\mathbf{v}_{8}$ and $\mathbf{v}_{k_{2}}=\mathbf{v}_{4}$. In this case, $\varphi=\varphi_{8}$ and $J_{1}\left(\varphi_{8}\right)=\{1\}, J_{2}\left(\varphi_{8}\right)=\{3,4,6,7\}, J_{3}\left(\varphi_{8}\right)=\{2\}, J_{4}\left(\varphi_{8}\right)=\{5,8\}$ and the statements given in Case 4 of the previous lemma for $\psi_{2}=\varphi_{4}$ and $\varphi=\varphi_{8}$ hold.

Next we are going to prove Statement (2) in Theorem 2.13, which will finish the proof of this result and our paper. Let $P_{j}$ be a point in $\mathcal{F}$ and assume $\mathbf{v}_{k}<\mathbf{v}_{j}$. Then, Lemma 3.12 proves that $\bar{\alpha}_{j} \geqslant \bar{\alpha}_{k}$ because the path $\left[\mathbf{v}_{k}, \mathbf{v}_{j}\right]$ is contained in a union of paths of the form $\left[\mathbf{v}_{j_{1}}, \mathbf{v}_{j_{2}}\right], \mathbf{v}_{j_{1}}$ and $\mathbf{v}_{j_{2}}$ being two consecutive vertices in $\mathcal{V} \cup \mathcal{V}_{\text {end }}$. Otherwise, a similar reasoning with the path $\left[\mathbf{v}_{j}, \mathbf{v}_{k}\right]$ also shows that $\bar{\alpha}_{j} \geqslant \bar{\alpha}_{k}$ and, by Proposition 2.3, the log-canonical threshold of $C$ is $\bar{\alpha}_{k}$.

It only remains to obtain the expression of $\bar{\alpha}_{k}$ when $P_{k} \in \mathcal{S}$ because otherwise Lemma 3.7 provides that expression. Let $C_{i_{1}}$ and $C_{i_{2}}$ be as in the statement and denote by $\varphi$ a 
curvette at $P_{k}$, then the following chain of equalities finishes the proof:

$\bar{\alpha}_{k}=\frac{c_{i_{1} i_{2}}+1}{\sum_{s=1}^{r} I\left(\varphi, f_{s}\right)}=\frac{c_{i_{1} i_{2}}+1}{c_{i_{1} i_{2}} \bar{\beta}_{0}^{i_{1}}+\sum_{i_{1} \neq s=1}^{r} c_{i_{1} i_{2}} \bar{\beta}_{0}^{s}}=\frac{\bar{\beta}_{0}^{i_{1}} \bar{\beta}_{0}^{i_{2}}+I\left(f_{i_{1}}, f_{i_{2}}\right)}{\bar{\beta}_{0}^{i_{1}} I\left(f_{i_{1}}, f_{i_{2}}\right)+\bar{\beta}_{0}^{i_{2}} \sum_{i_{1} \neq s=1}^{r} I\left(f_{i_{1}}, f_{s}\right)}$.

Notice that the second equality is a consequence of Lemma $3 \cdot 1$ and the last one has been obtained by multiplying numerator and denominator by $\bar{\beta}_{0}^{i_{1}} \bar{\beta}_{0}^{i_{2}}$.

Acknowledgements. Supported by Spain Ministry of Economy MTM2012-36917-C03-

03 and University Jaume I P1-1B2012-04.

\section{REFERENCES}

[1] M. Alberich-Carramiñana, J. Alvarez-Montaner and F. Dachs-Cadefau. Multiplier ideals in two-dimensional local rings with rational singularities. ArXiv:1412.3605.

[2] M. Alberich-Carramiñana, J. Alvarez-Montaner, F. Dachs-Cadefau and V. GonzÁlez-Alonso. Poincaré series of multiplier ideals in two-dimensional local rings with rational singularities. ArXiv:1412.3607.

[3] M. Aprodu and D. NAIE. Enriques diagrams and log-canonical thresholds of curves on smooth surfaces. Geom. Dedicata 146 (2010), 43-66.

[4] E. Artal Bartolo, P. Cassou-Nogués, I. Luengo and A. Melle-Hernández. On the log-canonical threshold for germs of plane curves. Contemp. Math. 474 (2008), 1-14.

[5] M.F. Aтiуah. Resolution of singularities and division of distributions. Comm. Pure Appl. Math. 23 (1970), 145-150.

[6] C. Birkar. Ascending chain condition for log-canonical thresholds and termination of log flips. Duke Math. J. 136 (2007), 173-180.

[7] C. Birkar, P. Cascini, C. Hacon and J. McKernan. Existence of minimal models for varieties of log general type. J. Amer. Math. Soc. 23 (2010), 405-468.

[8] E. Brieskorn and H. Knorrer. Plane Algebraic Curves, Birkhäuser, Basel, 1986.

[9] N. Budur. On Hodge spectrum and multiplier ideals. Math. Ann. 327 (2003), 257-270.

[10] N. Budur, P.D. GonzÁlez-PÉREz and M. GonzÁlez-Villa Log-canonical thresholds of quasi-ordinary hypersurface singularities. Proc. Amer. Math. Soc. 140 (2012), 4075-4083.

[11] N. Budur, M. Mustaţă and M. Saito. Bernstein-Sato polynomials of arbitrary varieties. Compos. Math. 142 (2006), 779-797.

[12] A. Campillo. Algebroid curves in positive characteristic, Lect. Notes Math. 613, SpringerVerlag, 1980.

[13] A. Campillo, F. Delgado and S.M. Gusein-Zade. On generators of the semigroup of a plane curve singularity. J. London Math. Soc. 60 (1999), 420-430.

[14] A. Campillo, G. González-Sprinberg and M. Lejeune-Jalabert. Clusters of infinitely near points. Math. Ann. 306 (1996), 169-194.

[15] F. Delgado. The semigroup of values of a curve singularity with several branches. Manuscripta Math. 59 (1987), 347-374.

[16] F. Delgado. An arithmetical factorization for the critical point set of some maps from $\mathbb{C}^{2}$ to $\mathbb{C}^{2}$, Singularities - Lille 1991, London Mathematical Society Lecture Note Series 201 (ed. Jean-Paul Brasselet), Cambridge University Press, 1994), 61-100.

[17] L. Ein, R. Lazarsfeld, K.E. Smith and D. VARolin. Jumping coefficients of multiplier ideals. Duke Math. J. 123 (2004), 469-506.

[18] C. Favre and M. Jonsson. Valuations and multiplier ideals. J. Amer. Math. Soc. 18 (2005), 655-684.

[19] C. Galindo and F. Monserrat. The Poincaré series of multiplier ideals of a simple complete ideal in a local ring of a smooth surface. Adv. Math. 225 (2010), 1046-1068.

[20] L.H. Halle and J. Nicaise. Motivic zeta functions of abelian varieties and the monodromy conjecture. Adv. Math. 227 (2011), 610-653.

[21] N. Hara, K.I. Yoshida. A generalization of tight closure and multiplier ideals. Trans. Amer. Math. Soc. 355 (2003), 3143-3174.

[22] E. HyRY and T. JÄRVILEHTO. Jumping numbers and ordered tree structures on the dual graph. Manuscripta Math. 136 (2011), 411-437.

[23] J. Igusa. Critical points of smooth functions and their normal forms, Complex and Algebraic Geometry, Iwanami Shoten, (1977), 357-368.

[24] T. JÄRVILEHTO. Jumping numbers of a simple complete ideal in a two-dimensional regular local ring, Mem. Amer. Math. Soc. 214 (2011), no. 1009. 
[25] J. Kollár. Singularities of pairs, Proc. Sympos. Pure Math. 62 (1997), 221-287.

[26] J. Kollár ET AL. Flips and abundance for algebraic threefolds, Astérisque 211 (1992).

[27] T. Kuwata. On log canonical thresholds of reducible curves. Amer. J. Math. 121 (1999), 701-721.

[28] R. Lazarsfeld. Positivity in Algebraic Geometry. Vol II, Springer, 2004.

[29] J. Lipman. Adjoints of ideals in regular local rings rings. Math. Res. Lett. 1 (1994), 739755, with an appendix by S.D. Cutkosky.

[30] J. Lipman and A. SAThAYE. Jacobian ideals and theorem of Briançon-Skoda about integral closures of ideals, Michigan Math. J. 28 (1981), 199-222.

[31] M. Mustaţă. Singularities of pairs via jet schemes. J. Amer. Math. Soc. 15 (2002), 599 615.

[32] D. NAIE. Jumping numbers of a unibranch curve on a smooth surface. Manuscripta Math. 128 (2009), 33-49.

[33] V. V. Shokurov. Three-dimensional log perestroikas (in Russian). With an appendix in English by Y. Kawamata, Izv. Ross. Akad. Nauk Ser. Mat. 56 (1992), 105-203, translation in Russian Acad. Sci. Izv. Math. 40 (1993), 95-202.

[34] K.E. Smith and H.M. Thompson. Irrelevant exceptional divisors for curves on a smooth surface. Contemp. Math. 448 (2007), 245-254.

[35] J.H.M. SteEnBRINK. The spectrum of hypersurface singularities. Astérisque 179-180 (1989), 163-184.

[36] K. TuCKER. Jumping numbers on algebraic surfaces with rational singularities. Trans. Amer. Math. Soc. 362 (2010), 3223-3241.

[37] K. TuCKER. Jumping numbers and multiplier ideals on algebraic surfaces, Ph. D. thesis, University of Michigan, 2010.

[38] A.N. VARCHENKO. Asymptotic Hodge structures in the vanishing cohomology. Math. USSR Izv. 18 (1982), 469-512.

[39] A.N. VARChEnKo. The complex singularity index does not change along the stratum $\mu=$ const (in Russian). Funktsional. Anal. i Prilozhen. 16 (1982), 1-12.

[40] A.N. Varchenko. Semicontinuity of the complex singularity exponent (in Russian). Funksional. Anal. i Prilozhen. 17 (1983), 77-78.

[41] O. Zariski and P. Samuel. Commutative Algebra. Vol II, Springer-Verlag, 1960. 